\title{
Automorphism Properties and Classification of Adinkras
}

\author{
B. L. Douglas, ${ }^{1}$ S. James Gates Jr., ${ }^{2}$ B. L. Segler, ${ }^{1}$ and J. B. Wang ${ }^{1}$ \\ ${ }^{1}$ School of Physics, University of Western Australia, Perth, WA 6009, Australia \\ ${ }^{2}$ Center for String and Particle Theory, Department of Physics, University of Maryland, College Park, MD 20742-4111, USA
}

Correspondence should be addressed to J. B. Wang; jingbo.wang@uwa.edu.au

Received 16 March 2015; Accepted 7 July 2015

Academic Editor: Pavel Kurasov

Copyright (C) 2015 B. L. Douglas et al. This is an open access article distributed under the Creative Commons Attribution License, which permits unrestricted use, distribution, and reproduction in any medium, provided the original work is properly cited.

\begin{abstract}
Adinkras are graphical tools for studying off-shell representations of supersymmetry. In this paper we efficiently classify the automorphism groups of Adinkras relative to a set of local parameters. Using this, we classify Adinkras according to their equivalence and isomorphism classes. We extend previous results dealing with characterization of Adinkra degeneracy via matrix products and present algorithms for calculating the automorphism groups of Adinkras and partitioning Adinkras into their isomorphism classes.
\end{abstract}

\section{Introduction}

Several recent studies [1-10] have introduced and developed a novel approach to the off-shell problem of supersymmetry. In particular, a graph theoretic tool has emerged to tackle this problem by encoding representations of supersymmetry into a family of graphs termed Adinkras. This graphical encoding has the advantage of allowing convenient manipulation of these objects, with the goal of achieving a deeper understanding of the underlying representations.

The classification of Adinkras is a natural goal of this work, and various aspects of this have been dealt with extensively in previous studies $[2,4,8,11]$, in which many of the properties of Adinkra graphs have been ascertained. The works of $[2,3]$ relate topological properties of Adinkras to doubly even codes and Clifford algebras. The work in [11] is particularly striking as apparently Betti numbers and functions similar to those of catastrophe theory seem on the horizon. However, we will not direct our current effort toward these observations.

The GAAC (Garden Algebra/Adinkra/Codes) Program began [12-15] with a series of observations of what appeared to be universal matrix algebra structures that seem to occur in all off-shell supersymmetrical theories. Two unexpected transformations have occurred since this start. First, there emerged Adinkras providing a graphical technology to represent these matrix algebras and second the connection of
Adinkras to codes. As the ultimate goal of this program is to provide a definitive classification of all off-shell supersymmetrical theories, the appearance of these new unexpected discoveries continues to be encouraging that the goal can be reached despite the pessimism surrounding this over thirtyyear-old unsolved problem.

The goal of this paper is to present a method of efficiently distinguishing Adinkras, in other words, a way of efficiently solving the graph isomorphism problem, restricted to this particular class of graphs. This is achieved via a classification of their automorphism group, together with an efficient algorithm for computing this automorphism group for any given Adinkra. In particular, we specify the automorphism group of an Adinkra in terms of its associated doubly even code. These codes are characterized in terms of local properties of Adinkras, such that the codes, and hence the associated equivalence and isomorphism classes of these graphs, can be efficiently computed.

The structure of the paper is as follows: Section 2 provides a formal graph theoretic definition of Adinkras, introducing some graph theoretic terms related to their study and discussing some basic properties that are derived from the definition. Section 3 defines the notions of equivalence and isomorphism on the class of Adinkras. We relate Adinkra graphs to doubly even codes, citing a result from [2] that all Adinkras have an associated code. The work of [3] relating properties of Adinkras to Clifford algebras is also discussed, 
and we define a standard form for Adinkras, used in the proof of later results. Section 4 establishes the main result of this work, classifying the automorphism group of valise Adinkras in terms of the related doubly even code. In Section 5 these results are used to generalize some results of [5] and provide a polynomial that partitions Adinkras into their equivalence classes. In Section 6 this is extended to isomorphism classes of nonvalise Adinkras, and we provide an associated algorithm that accomplishes this partitioning. Section 7 details some of the associated numerical methods and results and provides some additional examples.

\section{Adinkra Graphs}

2.1. Graph-Theoretic Notation. The Adinkra graphs dealt with in this work are simple, undirected, bipartite, edge- $N$-partite, and edge- and vertex-colored graphs. Note that in previous work [1-3] Adinkras are considered to be directed graphs. However, as this information is naturally encoded into the height assignment component of the vertex coloring, we remove the edge directions here to simplify the analysis.

We define a few of the graph theoretic terms below. For a more complete treatment, see [16].

A simple, undirected graph $G=(V, E)$ consists of a vertex set $V$ together with an edge set $E \subseteq V \times V$ of unordered pairs of $V$.

A graph is bipartite if its vertex set can be partitioned into two disjoint sets such that no edges lie wholly within either set. Equivalently, this is a graph containing no odd-length cycles.

A graph $G=(V, E)$ is edge-N-partite if its edge set $E$ can be partitioned into $N$ disjoint sets, such that every vertex $v \in V$ is incident with exactly one edge from each of these $N$ sets. In other words, a graph is edge- $N$-partite if it has a 1-factorisation.

Finally, a coloring of the edge or vertex set of a graph is a partitioning of these sets into different colour classes. Formally, this restricts the automorphism group of the graph to the subset that setwise stabilises these colour classes, the subset that does not map vertices (resp., edges) in one colour class to vertices (resp., edges) in another.

2.2. Definition and Properties. Adinkra graphs were introduced in [1] to study off-shell representations of supersymmetry. Thorough definitions are provided in $[2,3,8]$, although, as mentioned above, they vary slightly from the definition presented here in that for the purposes of this study we will consider them to be undirected graphs. The most current and complete definition of Adinkras can be found in Definition 3.2 in the work of [2]. With that in mind, we use the following definition of Adinkras for the remainder of this work.

Definition 1. An Adinkra graph $G=(V, E$, hgt, $\chi, \pi)$ is a bipartite, vertex- and edge-coloured graph with the following properties.

Each vertex in $V$ is coloured in two ways:

(i) a colouring $\chi: V \rightarrow \mathbb{Z}_{2}$ corresponding naturally to the bipartition (labelled as bosons and fermions); (ii) each vertex being given a height assignment, hgt: $V \rightarrow$ $\mathbb{Z}$ such that adjacent vertices are at adjacent heights.

The edges are also coloured in two ways:

(i) a partition into $N$ colour classes corresponding to an edge- $N$-partition of the graph;

(ii) an edge parity assignment $\pi: E \rightarrow \mathbb{Z}_{2}$; we term these two edge types dashed and solid.

Finally, the connections in the graph are essentially binary in the following manner. Every path of length two having edge colours $(i, j)$ defines a unique 4 -cycle with edge colours $(i, j, i, j)$. That is to say, of all 4 -cycles including these two edges, only one has the edge colours $(i, j, i, j)$. Such 4 -cycles all have an odd number of dashed edges.

We refer to the valence of an Adinkra as its dimension. Hence an Adinkra with $N$ edge colours (not counting edge parity) is an $\mathrm{N}$-dimensional Adinkra. There will generally be an assumed ordering of the edge colours from 1 to $N$, with the term $i$ 'th edges or $i$ 'th edge dimension referring to all edges of the $i$ 'th colour. The edge parity is often referred to as dashedness (see, e.g., [5]). In this work we also refer to it as the switching state of an edge or set of edges, motivated by a forthcoming analogy to switching and two graphs. Relative to the edge-colour ordering, the switching state of an edge $e=(u, v)$ of colour $i$ will be denoted alternately by $\pi_{i}(u), \pi_{i}(v)$, or $\pi(e)$ (referring to the $i$ 'th edge of the vertex $u$ or $v$ or simply the edge $e$ ).

The above conditions imply several additional properties. The edge- $N$-partite restriction requires equal numbers of bosons and fermions; hence the bipartition must consist of two sets of equal size. Moreover, we have the following important property.

Lemma 2. The number of vertices of any Adinkra is a power of two.

Proof. Since the Adinkra is $N$-regular and edge- $N$-partite and satisfies the 4-cycle property, there exists some $n \in$ $\mathbb{Z}^{+}, n \leq N$, such that, for any $n$-length subset of the edge colours (i.e., removing all of the edges whose colour does not lie within this subset), the Adinkra is simply an $n$ dimensional hypercube or $n$-cube. Hence we have that the number of vertices is equal to $2^{n}$. Note that if $n=N$, then ignoring edge and vertex colouring the resulting graph is simply the $N$-cube. Hence we term the corresponding Adinkra the $N$-cube Adinkra (in Section 4 we show that this is unique to $N)$. Where $n \neq N$, we denote the corresponding Adinkra to be an $(N, k)$ Adinkra, where $k=N-n$.

When drawing Adinkra graphs, we represent the bipartition by black and white vertices. As in previous work by Doran et al. [3], the height assignments will be represented by arranging the vertices in rows, incrementally, according to height.

Example 3. The Adinkras of Figure 1 satisfy all requirements listed above. Note that nodes in the same bipartition (bosons 


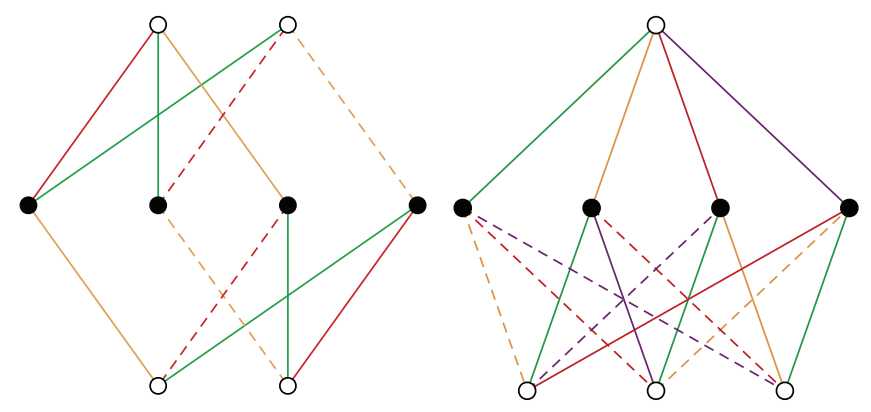

(i)

(ii)

FIgure 1: (i) A 3-cube Adinkra and (ii) a $(4,1)$ Adinkra.

or fermions) must be at the same height of modulo 2 and that every 4-cycle containing only two edge colours has an odd number of dashed/solid edges. In case (i), this is simply every 4-cycle; however case (ii) also contains 4-cycles with four edge colours.

It is also worth mentioning at this point that the questions of whether dashed edges correspond to even or odd parity, and which of the black or white node sets correspond to bosons or fermions, have been left ambiguous, as they do not impact on the following analysis and often represent a symmetry in the system. Also note that the absolute height values of height assignments are also currently ambiguous, as only relative values of height will be relevant to the following work. Finally, we note that since this work is concerned only with questions of equivalence and isomorphism of Adinkras, the assumption of connectedness will be made throughout to simplify the analysis, without loss of generality.

\section{Alternative Representations and Models of Adinkras}

There are other particularly useful ways of defining and modelling Adinkras. Before describing these it will be appropriate to introduce some more terminology, specifically relating to linear codes and Clifford algebras. We must also consider how to define notions of equivalence and isomorphism between Adinkras.

\subsection{Equivalence and Isomorphism Definitions}

Definition 4. Given an Adinkra ( $V, E$, hgt, $\chi, \pi)$, the topology of the Adinkra is defined as $(V, E)$, thus giving the underlying vertex and edge sets with all the colourings (including edge parity and height assignments) removed. When only the colourings associated with edge parity and height assignments are removed, the resulting graph $(V, E, \chi)$ is termed the chromotopology of the original Adinkra.

Two operations relative to a definition of equivalence have been defined on Adinkras [8]. The vertex lowering/raising operation consists of changing the height of a given set of vertices while preserving the requirement that adjacent vertices are at adjacent heights. Hence two $\operatorname{Adinkras}\left(V, E\right.$, hgt $\left._{1}, \chi, \pi\right)$ and $\left(V, E, \mathrm{hgt}_{2}, \chi, \pi\right)$ are related by a vertex lowering/raising operation if and only if $\forall u, v \in V,\left|\operatorname{hgt}_{1}(u)-\operatorname{hgt}_{1}(v)\right|=1 \Rightarrow$ $\left|\operatorname{hgt}_{2}(u)-\operatorname{hgt}_{2}(v)\right|$.

The operation of switching a vertex consists of reversing the parity of all edges incident with it. We note briefly that this preserves the property that 4-cycles with only two edge colours have an odd number of dashed edges.

This switching operation is analogous to Seidel switching of graphs (see $[17,18])$ in which adjacency and nonadjacency are swapped. In this case, adjacency has been replaced by parity/dashedness for the purposes of switching. Continuing this analogy, we define the switching class of an Adinkra $G$ to be the set of all Adinkras that can be obtained from $G$ by switching some subset of its vertices.

Any Adinkras in the same switching class will be considered isomorphic. Adinkras related via vertex raising/lowering operations will be considered equivalent, but not necessarily isomorphic.

Hence the definitions of equivalence and isomorphism considered here differ slightly. Permuting the vertex labels, switching and raising/lowering operations all preserve equivalence, whereas the only operations preserving isomorphism are those of switching and permutations of vertex labels.

Example 5. All three Adinkras drawn in Figure 2 are in the same equivalence class; however only the first two are isomorphic.

An automorphism of an $(N, k)$ Adinkra $G=(V, E$, hgt, $\chi, \pi)$ is a permutation of the vertex set, $p: V \rightarrow V, p \in$ $\operatorname{Sym}\left(2^{N-k}\right)$ that is also an isomorphism.

Note that as the ultimate goal of introducing Adinkras is a greater understanding of representations of off-shell supermultiplets, useful definitions of equivalence and isomorphism regarding Adinkras should be related to the underlying supermultiplets that they encode. However notions of isomorphism applied to supermultiplets are inherently contextspecific. A pertinent discussion relating to automorphisms of supermultiplets can be found in Appendix B of [6]. Here we cover a few of the points relating directly to Adinkras. In general, supermultiplets can be regarded as a triple $\left(Q, \mathbf{R}_{\phi}, \mathbf{R}_{\psi}\right)$, where $\mathbf{R}_{\phi}$ and $\mathbf{R}_{\psi}$ are vector spaces corresponding to the bosonic and fermionic component fields $\phi$ and $\psi$, and $Q=\left\{Q_{1}, \ldots, Q_{N}\right\}$ denotes the set of $N$ supercharges, or 


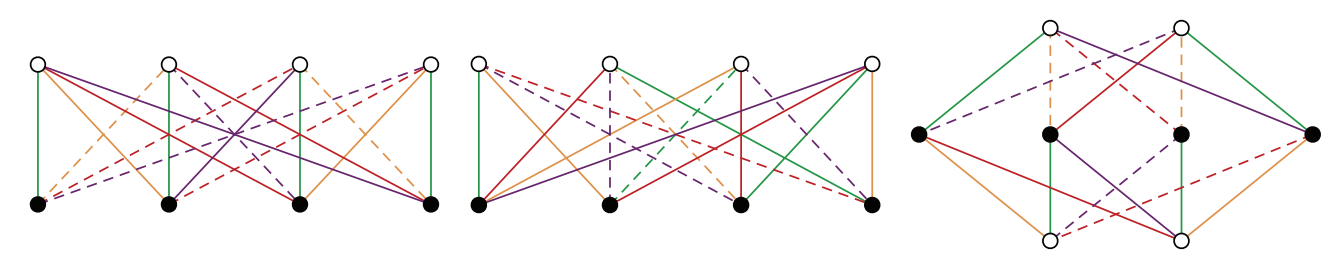

FIGURE 2: Three Adinkras belonging to the same equivalence class. The first two are isomorphic, whereas the third belongs to a separate isomorphism class.

supersymmetry transformation operators mapping between $\mathbf{R}_{\phi}$ and $\mathbf{R}_{\psi}$ and their derivatives. An Adinkra graph $G=$ $(V, E$, hgt, $\chi, \pi)$ can then be related to a supermultiplet $\mathscr{M}$, with each vertex in $V$ representing a component field of $\mathscr{M}$.

Transformations acting on a supermultiplet are grouped into two classes, inner and outer transformations. This distinction reflects the property that general supersymmetric models will consist of several supermultiplets, each consisting of distinct $\left(\mathbf{R}_{\phi}, \mathbf{R}_{\psi}\right)$ pairs whilst sharing the same set of supercharges $Q$. An inner transformation is one affecting a single supermultiplet while leaving the others unchanged. For instance, permuting the labels of the component fields of some $\left(\mathbf{R}_{\phi}, \mathbf{R}_{\psi}\right)$ pair affects only that supermultiplet. However a transformation of the set $\left\{Q_{1}, \ldots, Q_{N}\right\}$ affects all supermultiplets of a given supersymmetric model and is hence termed an outer transformation.

A transformation induces an automorphism when the result is indistinguishable from the original when its image and preimage are the same. However "indistinguishability" is inherently contextual, and so there are several notions of equivalence between supermultiplets. Whether an outer transformation induces an automorphism can depend on the particular model being studied; hence permutations of the Qlabels may or may not preserve equivalence.

Consequently, the definitions of equivalence and isomorphism provided above for Adinkras are not the only possible definitions, and as such a general classification of Adinkras must address a broader class of possible equivalences. One such alternative which is considered in Section 6.1 involves the inclusion of edge-colour permutations as an operation preserving isomorphism. As the edge colours (alternatively known as edge dimensions) correspond to the supercharges of the underlying supermultiplet, this situation simply involves allowing permutations of the set $\left\{Q_{1}, \ldots, Q_{N}\right\}$ to preserve isomorphism.

To introduce the tools necessary to classify the automorphism group properties of Adinkras, it will first be necessary to give a brief description of linear codes and Clifford algebras.

3.2. Linear Codes. A binary linear $(N, k)$ code $C \subseteq \mathbb{Z}_{2}^{n}$ consists of a set of $2^{k}$ elements, known as codewords, which form a subgroup of $\left(\mathbb{Z}_{2}^{n},+\right)$. Hence for all $u, v \in C, u+v \in C$. When dealing with a codeword $u$ of length $N$ we will use the notation $u=\left(u_{1}, u_{2}, \ldots, u_{N}\right)$, where $u_{i}$ is the $i$ th coordinate of $u$. When giving explicit examples of codewords, we will often omit the commas in this representation, as all coordinates are elements of $\mathbb{Z}_{2}$. We also consider all subsequent codes to be both binary and linear; these terms are henceforth dropped from their description.

A generating set of $C$ is a set $D \subseteq C$, consisting of $k$ codewords, such that all codewords in $C$ can be formed by a linear combination of elements of $D$. The minimal value of $k$ for which a generating set exists is called the dimension of the code and is invariant with respect to the particular choice of generating set.

The weight of a codeword $u$, denoted by wt $(u)$, or simply $|u|$, is the number of 1's in $u$. If every codeword in a code has a weight of $0(\bmod 4)$ the code is called doubly even. The inner product of two codewords $u$ and $v$ of length $N$ is defined as

$$
\langle u, v\rangle \equiv \sum_{i=1}^{N} u_{i} v_{i} \quad(\bmod 2)
$$

Then a doubly even code has the property that any two of its codewords have an inner product of 0 .

Definition 6. A standard form generating set $D$ of an $(N, k)$ code $C$ is defined to be of the form $D=(I \mid A)$, where $I$ is the $k \times k$ identity matrix and $A$ is a $k \times(N-k)$ matrix being the remainder of each codeword in $D$.

Example 7. By applying a permutation of the coordinates of codewords and considering an alternative generating set, the code $d_{8}$, generated by $\left(\begin{array}{llllllll}1 & 1 & 1 & 1 & 0 & 0 & 0 & 0 \\ 0 & 0 & 1 & 1 & 1 & 1 & 1 & 0 \\ 0 & 0 & 0 & 0 & 1 & 1 & 1 & 1 \\ 1\end{array}\right)$, can be written in terms of a standard form generating set. In order to do so, first we will interchange some coordinates by moving columns $C_{8} \rightarrow$

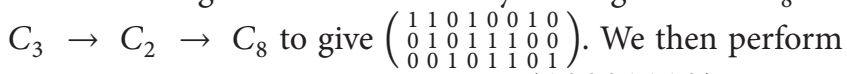
row operation $R_{1}+R_{2} \rightarrow R_{1}$ to give $\left(\begin{array}{llllllll}1 & 0 & 0 & 0 & 1 & 1 & 1 & 0 \\ 0 & 1 & 0 & 1 & 1 & 1 & 0 & 0 \\ 0 & 0 & 1 & 0 & 1 & 1 & 0 & 1\end{array}\right)$ which is in the standard form for a generating set.

Note that the freedom to interchange coordinates means that there are numerous standard form generating sets that we could produce, which in turn would have different codes associated with them. Given the nature of the standard form however, any code with an associated standard form generating set will have a unique standard form generating set.

Note also that generating sets will be assumed to be minimal in that the codewords comprising the set are linearly independent. Thus, a generating set with $n$ codewords corresponds to a code with $2^{n}$ distinct codewords.

Observation 1 . For any $(N, k)$ code, there exists a generating set $D$ and permutation of the coordinates of the codewords $p \in \operatorname{Sym}(N)$ such that $D$ is a standard form generating set. 


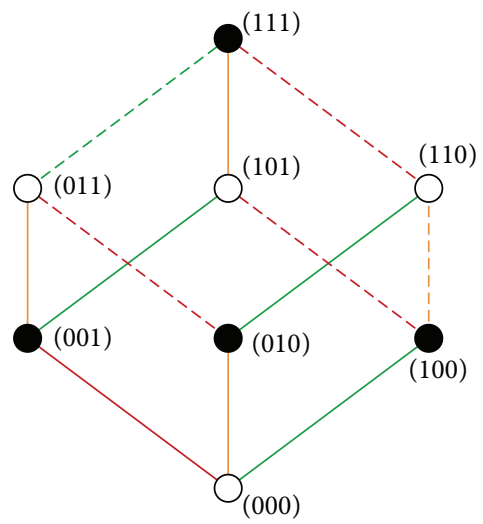

Figure 3: A 3-cube Adinkra shown together with a codeword representation for each vertex.

The notion of codewords can be effectively used to furnish a further organisation of the edge and vertex sets of an Adinkra. If we order the edge colours of an $N$-cube Adinkra from 1 to $N$, then assign each of these edge colours to the corresponding coordinate in an $N$-length codeword; each of the $2^{N}$ vertices in the Adinkra can be represented by such a codeword. These codewords are allocated in such a way that two vertices connected by the $i$ 'th edge differ precisely in the $i$ 'th element of their respective codewords.

Example 8. Applying this codeword representation to the vertices of a 3-cube Adinkra yields the graph of Figure 3. Note that, for an $N$-cube Adinkra, two vertices are connected by an edge if and only if their corresponding codeword representations have a Hamming distance of 1 (differing in only one position).

An important theorem of [2] links general $(N, k)$ Adinkras to doubly even codes. We paraphrase it, combined with other results from the same work, below.

Theorem 9 (see [2]). Every $(N, k)$ Adinkra (up to equivalence) class can be formed from the $N$-cube Adinkra by the process of quotienting via some doubly even $(N, k)$ code.

This quotienting process works as follows. We start with a doubly even $(N, k)$ code $C$ and an $N$-cube Adinkra with a corresponding edge-colour ordering and codeword representation. We then identify vertices (and their corresponding edges) related via any codeword in $C$, ensuring first that identified vertices have identical edge parities relative to the ordering of edge colours. In this process an $(N, k)$ code identifies groups of $2^{k}$ vertices and reduces the order of the vertex set from $2^{N}$ to $2^{N-k}$, producing an $(N, k)$ Adinkra. Doran et al. [2] showed that the topology of an Adinkra is uniquely determined by a doubly even code, with a 1-1 correspondence between the two.

Example 10. If we take the 4-cube Adinkra, with codewords given by the elements of $\mathbb{Z}_{2}^{4}$ and we quotient by the doubly even $(4,1)$ code $C=\{(0000),(1111)\}$, we produce the $(4,1)$ Adinkra of Figure 1. Quotienting by $C$ identifies pairs of codewords that differ by (1111) (e.g., (0000) and (1111); (0001) and (1110)), thus reducing the number of vertices from 16 to 8. We observe that this identification is consistent with the topology of the 4-cube Adinkra in that any edge joining two vertices $v_{1}$ and $v_{2}$ of the 4 -cube Adinkra can also be identified with an edge joining vertices $v_{1}+(1111)$ and $v_{2}+(1111)$ in the 4-cube Adinkra.

\subsection{Clifford Algebras}

Definition 11. One will consider the Clifford algebra $\mathrm{Cl}(n)$ to be the algebra over $\mathbb{Z}_{2}$ with $n$ multiplicative generators $\gamma_{1}, \gamma_{2}, \ldots, \gamma_{n}$, with the property

$$
\left\{\gamma_{i}, \gamma_{j}\right\}:=\gamma_{i} \gamma_{j}+\gamma_{j} \gamma_{i}=2 \delta_{i j} \mathbb{1}
$$

where $\delta_{i j}$ is the Kronecker delta. In other words, each of the generators is a root of $\mathbb{1}$, and any two generators anticommute.

It will be convenient to view the 4-cycle of Adinkras in terms of the anticommutativity property of Clifford generators. If we consider each position in the codeword representation of a vertex of an $N$-cube Adinkra to correspond to a given generator of $\mathrm{Cl}(N)$, with the vertex itself being related to the product of these Clifford generators, then the $i$ 'th edge dimension can be naturally viewed as the transformations corresponding to left Clifford multiplication by the $i$ 'th Clifford generator.

For example, a vertex with codeword representation (01100) corresponds to the product of Clifford generators $\gamma_{2} \gamma_{3} \in C l(5)$, and the edge connecting the vertex (01100) to (01101) is related to the mapping $\gamma_{2} \gamma_{3} \mapsto\left(\gamma_{5}\right)\left(\gamma_{2} \gamma_{3}\right)=$ $\gamma_{2} \gamma_{3} \gamma_{5}$. Then the condition that every 4-cycle which consists of exactly two edge colours must have an odd number of dashed edges corresponds to the anticommutativity property of Clifford generators, $\gamma_{i} \gamma_{j}=-\gamma_{j} \gamma_{i}$ for $i \neq j$.

In connection with this Clifford generator notation, we define a standard form for the switching state of an $N$-cube Adinkra.

Definition 12. A standard form $N$-cube Adinkra has edge parity as follows. The $i$ th edge of vertex $v=\left(v_{1}, v_{2}, \ldots, v_{N}\right)$ has edge parity given by 


$$
\pi_{i}(v) \equiv\left(v_{1}+v_{2}+\cdots+v_{i-1}\right) \quad(\bmod 2) .
$$

This corresponds to left Clifford multiplication of $v$ by $\gamma_{i}$. Note that this standard form is defined relative to some given codeword labelling of the vertex set. Since fixing the labelling of a single vertex fixes that of all vertices, we will sometimes refer to a standard form as being relative to a source node, being the vertex with label $(00 \cdots 0)$, or simply standard form relative to 0 .

Example 13. The 3-cube Adinkra of Example 8 is in standard form. Vertices are ordered into heights relative to their codewords, such that heights range from 0 (at the bottom) to 3 , and vertices at height $i$ have weight $i$. The edges are ordered from left to right, such that green corresponds to $\gamma_{1}$ and red corresponds to $\gamma_{3}$.

As we have seen, many Adinkras can be formed by quotienting the $N$-cube Adinkra with respect to some doubly even code (as we have not yet investigated the case of Gnomon Adinkras defined by the "zippering" process presented in [19], the extension of our results to these cases requires further study). However in practice this method can be quite inefficient. The following alternative method for constructing an $(N, k)$ Adinkra with associated code $C$ is due to $G$. Landweber (personal communication) is used by the Adinkramat software package:

(1) Start with the standard form $(N-k)$-cube Adinkra induced on the first $N-k$ edge dimensions.

(2) Find a standard form generating set for $C$, of the form $D=(I \mid A)$.

(3) Associate the $(N-k+i)$ th edge dimension with the product of Clifford generators given by $A_{i}$, the $i$ th row of $A$.

(4) The $i$ 'th edge connects a vertex $v$ to the vertex $v \cdot A_{i}$, with switching state corresponding to right Clifford multiplication of $v$ by $A_{i}$, up to a factor of $(-1)^{F}$.

The factor of $(-1)^{F}$, termed the fermion number operator, simply applies a $(-1)$ factor to fermions and leaves bosons unchanged. This factor is required to ensure the parity of an edge remains the same in either direction. Again, note that the choice of whether even- or odd-weight vertices are bosons remains ambiguous. For simplicity, we consider odd-weight vertices to be fermions for the purposes of the $(-1)^{F}$ factor, with the symmetry encoded by a graph-wide factor of \pm 1 in the switching state of the extra $k$ edge dimensions, corresponding to two potentially inequivalent $(N, k)$ Adinkras.

Define $\mathcal{S}_{\chi}(C)$ to be the set of all Adinkras obtained by the above construction method using the associated doubly even $(N, k)$ code $C$, with fermion/boson assignment determined by $\chi$. It remains to be seen whether $\delta_{\chi}(C)$ coincides with an equivalence class of $(N, k)$ Adinkras with associated code $C$. To our knowledge this has not been explicitly proven in previous work; hence we present a proof of this as follows.
Theorem 14. Given a doubly even $(N, k)$ code $C$ and a fermion/boson assignment function $\chi$, for any Adinkra $G \in \mathcal{S}_{\chi}(C)$ the equivalence class of $G$ coincides with $\mathcal{S}_{\chi}(C)$.

Proof. As we are only considering equivalence classes, the height assignments will be ignored.

Consider an $N$-cube Adinkra quotiented with respect to an $(N, k)$ code $C$ with standard form generating set $D=$ $(I \mid A)$, producing an $(N, k)$ Adinkra $G=(V, E$, hgt, $\chi, \pi)$. Assume without loss of generality that the vertices remaining after quotienting all have fixed $(N-k+1)$ th $\rightarrow N$ th codeword characters (e.g., all fixed as 0 ). Then the rows of $A$ represent the vertices identified by the quotienting process. We want to show that, up to isomorphism, there are at most two different ways this quotienting operation can be performed.

Isolating any set of $N-k$ edge dimensions of $G$ yields an induced $(N-k)$-cube Adinkra, $H=\left(V, E^{\prime}\right.$, hgt, $\left.\chi, \pi\right)$, with $E^{\prime} \subset E$ and $\pi$ restricted to $E^{\prime}$. We will see in Section 4 that, for fixed $m$, all $m$-cube Adinkras belong to a single equivalence class. Hence without loss of generality $H$ can be considered to be in standard form. This in turn fixes the switching state of all edges belonging to the chosen $N-k$ edge dimensions. Now only a single degree of freedom remains in the switching states of the remaining $k$ edge dimensions, due to the 4 cycle condition. In other words, fixing the switching state of any one of the remaining edges fixes all remaining edges. This choice corresponds to a graph-wide factor of \pm 1 in the switching state of the additional $k$ edge dimensions and is determined by $\chi$.

Since this graph-wide factor of \pm 1 matches the difference between the two possibly inequivalent Adinkras produced by the above construction method, it remains to show that the construction method does indeed produce valid $(N, k)$ Adinkras. Hence me must verify that the 4 -cycle condition holds.

As odd-weight vertices are considered to be fermions, the $(-1)^{F}$ factor can be replaced by an additive factor of $\sum_{j=1}^{N-k} x_{j}$, for a given vertex $x \in V$. Then for $i>N-k, x \in V, A_{i}=$ $\left(a_{1}, a_{2}, \ldots, a_{N-k}\right)$

$$
\begin{aligned}
\pi_{i}(x) \equiv & \sum_{j=1}^{N-k} x_{j}+x \cdot A_{i} \\
\equiv & \sum_{j=1}^{N-k} x_{j}+x_{2}\left(a_{1}\right)+x_{3}\left(a_{1}+a_{2}\right)+\cdots \\
& +x_{N-k}\left(a_{1}+a_{2}+\cdots+a_{(N-k-1)}\right) \quad(\bmod 2) .
\end{aligned}
$$

The 4-cycles in $G$ with two edge colours $i$ and $j$ will be split into three cases:

(i) $i, j \leq N-k, \quad i<j$,

(ii) $i \leq N-k, j>N-k$,

(iii) $i, j>N-k$.

Note that a given vertex $x \in V$ defines a unique such 4 -cycle. In case (i), consider two antipodal points of such a 4 -cycle, $x$ 


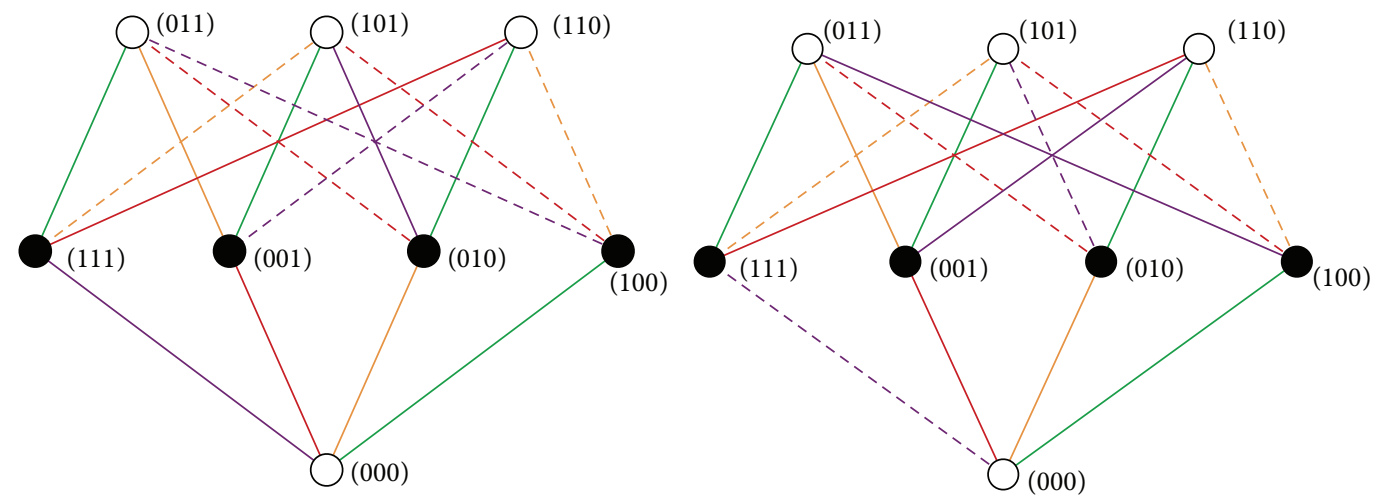

FIgURE 4: Two $(4,1)$ Adinkras with switching state in standard form.

and $x+i+j$. Then the sum of the edge parities of the 4-cycle equals

$$
\begin{aligned}
& \left(x_{1}+\cdots+x_{i-1}\right)+\left(x_{1}+\cdots+x_{j-1}\right)+\left(x_{1}+\cdots+x_{i-1}\right) \\
& +\left(x_{1}+\cdots+\left(x_{j}+1\right)+\cdots+x_{j-1}\right) \equiv 1 \quad(\bmod 2) .
\end{aligned}
$$

Hence the 4-cycle condition holds (note that this is the only case present in the construction of the standard form $N$-cube Adinkra).

For case (ii), the antipodal points are $x$ and $z$, where

$$
\begin{aligned}
z & \equiv\left(x_{1}+a_{1}, \ldots, x_{i}+a_{i}+1, \ldots, x_{N-k}+a_{N-k}\right) \\
& \equiv x+A_{j}+i \quad(\bmod 2),
\end{aligned}
$$

and by substituting (4), the sum becomes

$$
\begin{gathered}
\left(x_{1}+\cdots+x_{i-1}\right)+\left(\sum_{l=1}^{N-k} x_{l}+x \cdot A_{j}\right) \\
+\left(x_{1}+\cdots+x_{i-1}\right)+\left(a_{1}+\cdots+a_{i-1}\right) \\
+\left(\sum_{l=1}^{N-k} z_{l}+z \cdot A_{j}\right) \quad(\bmod 2) .
\end{gathered}
$$

However $\left|A_{j}\right| \equiv 3(\bmod 4)$, so $|x| \equiv|z|(\bmod 2)$. Then the first and third terms cancel, as do the second and fifth, leaving

$$
\begin{aligned}
x \cdot A+ & z \cdot A+\left(a_{1}+\cdots+a_{i-1}\right) \\
\equiv & 2(x \cdot A)+2\left(a_{1}+\cdots+a_{i-1}\right)+a_{2}\left(a_{1}\right) \\
& +a_{3}\left(a_{1}+a_{2}\right)+\cdots+a_{N-k}\left(a_{1}+\cdots a_{(N-k-1)}\right) \\
\equiv & \left|A_{j}-1\right|+\left|A_{j}-2\right|+\cdots+1 \equiv \frac{1}{2}|A-1||A|
\end{aligned}
$$

$(\bmod 2)$

Now $|A| \equiv 3(\bmod 4) ;$ hence

$$
\begin{aligned}
\frac{1}{2}|A-1||A| & \equiv 3 \quad(\bmod 4) \\
& \equiv 1 \quad(\bmod 2),
\end{aligned}
$$

and case (ii) is verified.
Case (iii) proceeds similarly to case (ii).

Hence the graphs produced via this construction method are indeed $(N, k)$ Adinkras. Since [2] showed that all $(N, k)$ Adinkras can be produced via the quotienting method, there can be at most two equivalence classes of Adinkras with the same associated code, and hence the Adinkras produced from the two methods must coincide.

Definition 15. A standard form $(N, k)$ Adinkra has switching state given by the above construction, relative to an associated doubly even code.

Example 16. The smallest nontrivial $(N, k)$ Adinkra has parameters $N=4, k=1$, with associated doubly even code (1111). The standard form of Definition 15 is shown in Figure 4, for each choice of the graph-wide factor of \pm 1 .

In this case the two Adinkras formed belong to different equivalence classes, as we demonstrate in the following section.

This construction provides a much simpler practical method of constructing Adinkras, and the standard form will be convenient in the proof of later results. Note that all $(N, k)$ Adinkras have an associated doubly even code, with matching parameters of length and dimension. For these purposes the $N$-cube Adinkra is considered an $(N, 0)$ Adinkra with a trivial associated code of dimension 0 .

\section{Automorphism Group Properties of Adinkras}

We now have sufficient tools to derive the main result of this part: classifying the automorphism group of an Adinkra with respect to the local parameters of the graph, namely, the associated doubly even code. We start with several observations regarding the automorphism properties of Adinkras.

Lemma 17. Ignoring height assignments, the N-cube Adinkra is unique to $N$, up to isomorphism.

Note that equivalently, we may consider Adinkras with only two heights to correspond to the assignment of bosons and fermions, and we call these valise Adinkras. Those with more than two heights are called nonvalise Adinkras. 
Proof. It suffices to show that any $N$-cube Adinkra can be mapped to standard form via some set of vertex switching operations. Such a mapping can be trivially constructed. For example, start with a single vertex. The parity of its edge set can be mapped to any desired form by a set of vertex switching operations applied only to its neighbours. In particular the edge parities can be mapped to those of the corresponding standard form Adinkra. Since the 4-cycle restriction of Section 2.2 holds, this process can be continued consistently for the set of vertices at each subsequent distance from this original vertex. For example, there will be ${ }^{N} C_{2}$ vertices at a distance of two from the original vertex and each of these vertices will belong to one of the ${ }^{N} C_{2}$ unique 4 -cycles defined by the pairs of edges joining the original vertex to its neighbours. For each of these 4-cycles, since we have already fixed two of its edges to have the correct parity (those joining the original vertex to its neighbours), the 4cycle property (that the cycle must contain an odd number of dashed edges) ensures that the remaining two edges both must either have the correct parity (requiring no vertex switching) or have incorrect parity (which can be rectified by switching the vertex). This process can then be continued in the same manner for vertices at further distances. The Adinkra resulting from this process will then be in standard form relative to this original vertex, regardless of the initial switching state of the Adinkra.

Lemma 18. $N$-cube valise Adinkras are vertex-transitive up to the boson/fermion bipartition. That is to say, given any two vertices $v_{1}$ and $v_{2}$ of the $N$-cube Adinkra, there exists an automorphism $\gamma$ which maps $v_{1} \rightarrow v_{2}$.

Proof. To show this, we begin with a standard form $N$-cube Adinkra $G=(V, E$, hgt, $\chi, \pi)$, with switching state defined by left multiplication by the corresponding Clifford generators, as in Definition 12. Since $N$-cube Adinkras are unique up to isomorphism, in the sense of Lemma 17, this can be done without loss of generality.

Then the switching state of edge $(x, y)$, where $x=i \cdot y$, is of the form $x_{1}+x_{2}+\cdots+x_{i-1}(\bmod 2)$. Consider a nontrivial permutation $\rho: V \mapsto V$. Since $\rho$ is nontrivial, there are two vertices $u, v \in V, u \neq v$, such that $\rho: u \mapsto v$. If $\rho$ is to preserve the topology of $G$, we must have $\rho: x \mapsto(u \cdot v) x$, $\forall x \in V$. Note that $\rho$ has no fixed points and is completely defined in terms of $(u \cdot v)$. Any automorphism $\gamma$ of $G$ which permutes $V$ according to $\rho$ must also switch some set of vertices in such a way that edge parity is preserved. However $\rho$ maps the edge $(x, y)$, where $x=i \cdot y$, with edge parity $x_{1}+x_{2}+\cdots+x_{i-1}(\bmod 2)$, to the edge $(\rho(x), \rho(y))$, with edge parity of

$$
\begin{aligned}
& \left(x_{1}+x_{2}+\cdots+x_{i-1}\right) \\
& \quad+\left(\left(u_{1}+v_{1}\right)+\left(u_{2}+v+2\right)+\cdots+\left(u_{i-1}+v_{i-1}\right)\right)
\end{aligned}
$$

$(\bmod 2)$.

Hence the change in edge parity of $(x, y)$ does not depend on either endpoint explicitly, only on the mapping $\rho$ and the edge dimension $i$. In other words, $\rho$ either preserves the switching state of all edges of a given edge dimension or reverses the parity of all such edges. Note that any single edge dimension can be switched (while leaving all other edges unchanged) via a set of vertex switching operations; hence an automorphism $\gamma$ exists for all such $\rho$. Furthermore, $\gamma$ is unique to $\rho$, which is in turn unique to the choice of $(u \cdot v)$.

Note that if $G$ is in standard form, we term the automorphism mapping vertex $x$ to vertex $0=(00 \cdots 0)$ as putting $G$ in standard form relative to $x$. By this terminology, $G$ was initially in standard form relative to 0 .

Corollary 19. The $N$-cube Adinkra is minimally vertextransitive (up to the bipartition), in the sense that the pointwise stabiliser of the automorphism group is the identity, and $|\operatorname{Aut}(G)|=(1 / 2)|V|=2^{N-1}$. In other words, no automorphisms exist that fix any points of the $N$-cube Adinkra.

Consider any $(N, k)$ Adinkra $G=(V, E$, hgt, $\chi, \pi)$. The sub-Adinkra $H=\left(V, E^{\prime}\right.$, hgt, $\left.\chi, \pi\right)$ induced on any set $S$ of $(N-k)$ edge dimensions of $G$ will be an $(N-k)$-cube Adinkra. Also, for all such $S$ there exists some set of vertex switching operations such that the induced Adinkra $H$ is in standard form. In the case where $S=(1,2, \ldots, N-k)$, the induced $H$ is in standard form if and only if $G$ is in standard form.

Observation 2. Given some $(N, k)$ Adinkra, containing a standard form $(N-k)$-cube Adinkra induced on the first $(N-k)$ edge dimensions (or equivalently an induced $(N-k)$ cube Adinkra in any given form), the switching state of the $i$ 'th edge dimension, $i>(N-k)$, is fixed up to a graph wide factor of -1 .

In particular, fixing the parity of the extra edges $i$, where $i>(N-k)$, of a single vertex fixes the parity of all extra edges in the graph. This is a direct consequence of the anticommutativity property of 4 -cycles with two edge colours.

This reduces the problem of finding automorphisms of a general $(N, k)$ Adinkra to that of local mappings between vertices. In particular, for any two nodes $x, y \in V$, there will be a unique automorphism of the induced $(N-k)$ cube Adinkra mapping $x$ to $y$. This will extend to a full automorphism of $G$ if and only if it preserves the switching state of the additional $k$ edges of $x$. Hence we arrive at the following result.

Theorem 20. Consider an $(N, k)$ Adinkra $G=(V, E$, hgt, $\chi, \pi)$ having an associated code $C$. Two vertices $x, y \in$ $V$ are equivalent (disregarding vertex colouring, there is an automorphism mapping between them) if and only if their relative inner products with respect to each codeword in $C$ are equal. In other words, $\exists \gamma \in \operatorname{Aut}(G)$ such that $\gamma: x \mapsto y$ iff $\forall c \in C,\langle x, c\rangle \equiv\langle y, c\rangle(\bmod 2)$.

Proof. Consider without loss of generality the case where $G$ is in standard form, and take $H=\left(V, E^{\prime}\right.$, hgt, $\left.\chi, \pi\right)$ to be the sub-Adinkra induced on the first $N-k$ edge dimensions. By Lemma $18, H$ is vertex transitive if we disregard vertex colouring. So consider the automorphism $\rho$ of $H, \rho: x \mapsto y$, where $x, y \in V$. Now $\rho$ consists of two parts: a permutation 
of the vertex set, $\rho: v \mapsto x \cdot y \cdot v, \forall v \in V$, and a set of vertex switching operations, such that the switching state of $H$ is preserved.

We wish to know when $\rho$ switches $x$ relative to $y$ (i.e., when $x$ is switched but $y$ is not, or vice versa, and when either both or neither are switched). Where $\pi_{i}(x)$ denotes the switching state of the $i$ 'th edge of $x$ (in $G$ or $H$ ), we use $\pi_{i}^{\prime}(x)$ to denote the equivalent switching state of $x$ in $\rho(G)$ or $\rho(H)$ (we also denote $\rho(G)$ and $\rho(H)$ by $G^{\prime}$ and $H^{\prime}$, resp.). Note that $x$ in $G^{\prime}$ is the image of $y$ in $G$ under $\rho$.

For edge dimension $i \leq N-k$, we see that since $\rho$ is an automorphism of $H, \pi_{i}(x)=\pi_{i}^{\prime}(y)$, and similarly $\pi_{i}(v \cdot x)=$ $\pi_{i}^{\prime}(v \cdot y) \forall v \in V$. For the case $i>N-k, \pi_{i}(v)$ is given by (4) in the proof of Theorem 14, but what about $\pi_{i}^{\prime}(v)$ ?

The value of $\pi_{i}^{\prime}(v)$ can be found by considering the path $P_{i}(x)$ in $H$ joining $x$ to $x \cdot A_{i}$, where $\mathrm{A}_{i}=\left(a_{1}, a_{2}, \ldots, a_{N-k}\right)$. If we denote by $A_{i}$ the associated product of Clifford generators

$$
A_{i}=\prod_{j=1}^{n} \gamma_{s_{j}}
$$

where $s_{j}$ denote the 1's of $A_{i}$, we have

$$
P_{i}(x)=\left(x, x \cdot a_{s_{1}},\left(x \cdot a_{s_{1}}\right) \cdot a_{s_{2}}, \ldots, x \cdot A_{i}\right) .
$$

Then $\rho$ will preserve the parity of edge $\left(x, x \cdot A_{i}\right)$ if and only if the number of dashed edges in paths $P_{i}(x)$ and $P_{i}(y)$ are equal $(\bmod 2)$. In other words,

$$
\pi_{i}(x)=\pi_{i}^{\prime}(x) \text { iff } \sum_{e \in P_{i}(x)} \pi(e) \equiv \sum_{e \in P_{i}(y)} \pi(e)
$$

$(\bmod 2)$.

Now $\pi_{s_{1}}(x) \equiv x_{1}+\cdots+x_{s_{1}-1}(\bmod 2), \ldots, \pi_{s_{n}}\left(x \cdot A_{i}\right) \equiv$ $\left(x_{1} a_{1}\right)+\cdots+\left(x_{s_{n}} a_{s_{n}}\right)(\bmod 2)$, where $a_{i}=1$ for $i \in\left\{s_{j}: 1 \leq\right.$ $j \leq n\}$, and $a_{i}=0$ elsewhere. Hence this can be rearranged as

$$
\begin{aligned}
\sum_{e \in P_{i}(x)} \pi(e) \equiv & a_{2}\left(x_{1}\right)+a_{3}\left(x_{1}+x_{2}\right)+\cdots \\
& +a_{N-k}\left(x_{1}+\cdots+x_{N-k-1}\right) \\
\equiv & x_{1}\left(a_{2}+\cdots+a_{N-k}\right)+\cdots \\
& +x_{N-k-1}\left(a_{N-k}\right) \quad(\bmod 2) .
\end{aligned}
$$

Hence, $\pi_{i}(x) \equiv|x|+x \cdot A_{i}(\bmod 2)$, and $\pi_{i}^{\prime}(y) \equiv \pi_{i}(x)+$ $\left(\sum_{e \in P_{i}(x)} \pi(e)-\sum_{e \in P_{i}(y)} \pi(e)\right)$, and note that $\sum_{e \in P_{i}(x)} \pi(e)+x \cdot A_{i}$ simplifies to

$$
\left|A_{i}\right||x|-\sum_{j=1}^{N-k}\left(x_{j} a_{j}\right),
$$

so we have, for $i>N-k$,

$$
\begin{aligned}
\pi_{i}(x)-\pi_{i}^{\prime}(y) \equiv & |x|+\left|A_{i}\right||x|+|y|+\left|A_{i}\right||y| \\
& +\sum_{j=1}^{N-k}\left(x_{j} a_{j}\right)+\sum_{j=1}^{N-k}\left(y_{j} a_{j}\right)
\end{aligned}
$$

$(\bmod 2)$.
Since $\left|A_{i}\right| \equiv 1(\bmod 2)$, we have $|x|\left(\left|A_{i}\right|+1\right) \equiv 0(\bmod 2)$, and the first 4 terms cancel, leaving

$$
\begin{aligned}
\pi_{i}(x)-\pi_{i}^{\prime}(y) & \equiv \sum_{j=1}^{N-k}\left(x_{j} a_{j}\right)+\sum_{j=1}^{N-k}\left(y_{j} a_{j}\right) \\
& \equiv\left\langle x, A_{i}\right\rangle+\left\langle y, A_{i}\right\rangle \quad(\bmod 2),
\end{aligned}
$$

which completes the proof.

Observation 3. Two vertices having the same relative inner products with respect to each element of a set $S$ of codewords also have the same relative inner product with respect to the group generated by $S$, under addition modulo 2 .

Corollary 21. Conversely to Theorem 20, consider a single orbit $\phi$ of the $(N, k)$ Adinkra $G$ with associated doubly even code $C$. If all elements of $\phi$ have fixed inner product relative to an $N$-length codeword $c$, then $c \in C$, the code by which $G$ has been quotiented.

Theorem 20 leads to several important corollaries regarding the automorphism group properties of Adinkras. Recall that there are at most two equivalence classes of Adinkras with the same associated doubly even code. Theorem 20 implies that, disregarding the vertex colourings, there is in fact only one such equivalence class. Hence including the vertex bipartition, we see that two equivalence classes exist if and only if the respective vertex sets of bosons and fermions are setwise nonisomorphic. This in turn occurs if and only if at least one orbit (and hence all orbits) of $G$ is of fixed weight modulo 2 .

In $[2,5]$, one such case was investigated, for Adinkras with parameters $N=4, k=1$. In this case, the Klein flip operation, which exchanges bosons for fermions, was found in $[2,5]$ to change the equivalence class of the resulting Adinkra. We term this property Klein flip degeneracy and note the following corollary of Theorem 20.

Corollary 22. An $(N, k)$ Adinkra with associated code $C$ has Klein flip degeneracy if and only if the all-1 codeword $(11 \cdots 1) \in C$. This in turn occurs only if $N \equiv 0(\bmod 4)$.

Corollary 23. The automorphism group of a valise $(N, k)$ Adinkra $G=(V, E$, hgt, $\chi, \pi)$ has size $2^{N-2 k-a}$, with $2^{k+a}$ orbits of equal size, where $a=0$ if $C$ contains the all-1 codeword, and $a=1$ otherwise.

Proof. $G$ has $2^{N-k}$ nodes. By Theorem 20, disregarding the vertex bipartition there are $2^{k}$ orbits of equal size, partitioning the vertex set. Including the bipartition, these orbits are split into 2 once more whenever they contain nodes of variable weight modulo 2 (i.e., whenever $C$ does not contain the all-1 codeword).

\section{Characterising Adinkra Degeneracy}

In the work of Gates et al. [5] the Klein flip degeneracy in the $N=4$ case of Example 16 was investigated. It was shown 
that Adinkras belonging to these two equivalence classes can be distinguished via the trace of a particular matrix derived from each Adinkra. We will briefly introduce these results and generalise them to general $(N, k)$ Adinkras.

Throughout the following section we will consider an $(N, k)$ Adinkra $G=(V, E$, hgt, $\chi, \pi)$ with corresponding code $C$. Note that $G$ has $2^{N-k}$ vertices, each of degree N.

\subsection{Notation}

Definition 24. The adjacency matrix $A(G)$ (or simply $A$ where the relevant Adinkra is clear from the context) is a $2^{N-k} \times$ $2^{N-k}$ symmetric matrix containing all the information of the graphical representation, except the vertex colouring associated with height assignments. Each element of the main diagonal represents either a boson or a fermion, denoted by $+i$ for bosons and $-i$ for fermions. The offdiagonal elements represent edges of $G$, numbered according to edge dimension, with sign denoting dashedness. For example, if position $A_{x, y}=-4$, there is a dashed edge of the fourth edge colour between boson/fermion $x$ and fermion/boson $y$. Hence the sign of the main diagonal elements represents the vertex bipartition, the sign of offdiagonal elements represents the edge parity, the absolute value of off-diagonal elements represents edge colour, and the position of the elements encodes the topology of the Adinkra.

Example 25. Consider the 3-cube Adinkra of Example 8.

If we order the vertex set into bosons and fermions, this Adinkra can be represented by the matrix:

$$
A=\left[\begin{array}{cccccccc}
i & 0 & 0 & 0 & 1 & 2 & 3 & 0 \\
0 & i & 0 & 0 & -2 & 1 & 0 & 3 \\
0 & 0 & i & 0 & -3 & 0 & 1 & -2 \\
0 & 0 & 0 & i & 0 & -3 & 2 & 1 \\
1 & -2 & -3 & 0 & -i & 0 & 0 & 0 \\
2 & 1 & 0 & -3 & 0 & -i & 0 & 0 \\
3 & 0 & 1 & 2 & 0 & 0 & -i & 0 \\
0 & 3 & -2 & 1 & 0 & 0 & 0 & -i
\end{array}\right] .
$$

Note that the symmetric nature of the adjacency matrix arises naturally out of the definition, and requiring that this property be upheld imposes no further restrictions in and of itself.

We define $L$ and $R$ matrices similarly, as in [5], to represent a single edge dimension of the Adinkra. $L$ and $R$ matrices encode this edge dimension, with rows corresponding to bosons and fermions, respectively. As each edge dimension is assigned a separate matrix, the elements corresponding to edges are all set to \pm 1 . Then the Adinkra of Example 25 has $L$ matrices:

$$
\begin{aligned}
L_{1} & =\left[\begin{array}{llll}
1 & 0 & 0 & 0 \\
0 & 1 & 0 & 0 \\
0 & 0 & 1 & 0 \\
0 & 0 & 0 & 1
\end{array}\right], \\
L_{2} & =\left[\begin{array}{cccc}
0 & 1 & 0 & 0 \\
-1 & 0 & 0 & 0 \\
0 & 0 & 0 & -1 \\
0 & 0 & 1 & 0
\end{array}\right],
\end{aligned}
$$

$$
L_{3}=\left[\begin{array}{cccc}
0 & 0 & 1 & 0 \\
0 & 0 & 0 & 1 \\
-1 & 0 & 0 & 0 \\
0 & -1 & 0 & 0
\end{array}\right] \text {. }
$$

These matrices can be read directly off the top right quadrant of the adjacency matrix. Similarly, the $R$ matrices can be read off the lower left quadrant. In this case, we have $R_{i}=$ $L_{i}^{T}$, where $T$ denotes the matrix transpose. Note that $L$ and $R$ matrices taken directly from the adjacency matrix will always be related via matrix transpose. Furthermore, since we are considering only off-shell supermultiplets, these matrices must also be square. We will assume that all subsequent $L$ and $R$ matrices are related in this way. We define a further object, $\gamma_{\mathrm{i}}$, to be a composition of $L$ and $R$ matrices of the form

$$
\gamma_{i}=\left[\begin{array}{cc}
0 & L_{i} \\
R_{i} & 0
\end{array}\right] .
$$

Further details regarding these objects can be found in [5]. In particular, it was shown in [5] that the Klein flip degeneracy of several $(4,1)$ Adinkras can be characterised by the trace of quartic products of these matrices, according to the formula

$$
\begin{aligned}
& \operatorname{Tr}\left(L_{i}\left(L_{j}\right)^{T} L_{k}\left(L_{l}\right)^{T}\right) \\
& \quad=4\left(\delta_{i j} \delta_{k l}-\delta_{i k} \delta_{j l}+\delta_{i l} \delta_{j k}+\chi_{0} \epsilon_{i j k l}\right),
\end{aligned}
$$

where $\delta$ represents the Kronecker delta and $\epsilon$ the Levi-Civita symbol and where $\chi_{0}= \pm 1$ distinguishes between the $(4,1)$ Adinkras belonging to the two equivalence classes of Example 16. It is also conjectured in [5] that this same method for distinguishing equivalence classes can be generalised to all values of the parameters $N$ and $k$. We prove this conjecture in the following section.

5.2. Degeneracy for General N. In order to generalise (21) to higher $N$, we wish to determine the form taken by the trace of products of these $\gamma$ matrices and establish exactly when this can be used to partition Adinkras into their equivalence classes. Firstly we note the following properties of Adinkras.

Lemma 26. The cycles of an N-cube Adinkra consist entirely of paths containing each edge dimension $0(\bmod 2)$ times. 
Lemma 27. The cycles of an $(N, k)$ Adinkra with associated code $C$ consist of paths in which

(i) each edge dimension is traversed $0(\bmod 2)$ times;

(ii) at least one edge dimension is traversed $1(\bmod 2)$ times.

In case (ii), the set of such edge dimensions traversed $1(\bmod 2)$ times corresponds to a codeword in $C$.

In fact, the codeword $(00 \cdots 0)$ is in any such code $C$, so all cycles have this property; however case (i) is considered a trivial instance.

Consider the product of $t \gamma$ matrices of $G$, denoted by $M=\gamma_{i_{1}} \gamma_{i_{2}} \cdots \gamma_{i_{t}}$. As we are considering only $L$ and $R$ matrices such that $L_{i}=R_{i}^{T}$, all $\gamma$ matrices defined as in (20) will be real and symmetric. This leads to the following property for $M$.

Lemma 28. The elements of the main diagonal of $M$ are nonzero if and only if the path $P_{M}=\left(i_{1}, i_{2}, \ldots, i_{t}\right)$ represents a closed loop within the Adinkra. This can occur by one of two ways:

(i) trivially, if each edge dimension contained in $p$ is present $0(\bmod 2)$ times,

(ii) if p corresponds to a codeword (or set of codewords) in C.

Proof. Consider the case $P_{M}=(i, j)$. If $i=j, M$ is trivially the identity, and hence (i) holds. If $i \neq j$, then $M_{x, y} \neq 0$ if and only if vertices $x$ and $y$ are connected via a path $(i, j)$ (i.e., if $x=i \cdot j \cdot y$ ). Extending to general $M$, if $M_{x, x} \neq 0$ for some $x \in V$, this implies that $x$ is connected to itself via the path $P_{M}$, a closed loop/cycle in $G$. Hence Lemma 27 completes the proof.

Corollary 29. $M_{x, x} \neq 0$ for some $x \in V$ if and only if this is true for all $x \in V$.

Lemma 30. In case (ii) of Lemma 28, with $M_{x, x}= \pm 1$ for all $x \in V, M_{x, x}=M_{y, y}$ if and only if $x$ and $y$ have the same inner product with respect to the codeword corresponding to $P_{M}$.

Proof. Consider the product of Clifford generators corresponding to path $P_{M}$. Since (ii) holds, after cancelling repeated elements we are left with some codeword $p \in C$, such that $p=a P_{M}$, where $a= \pm 1$, depending on whether $P_{M}$ corresponds to an even or odd permutation of $p$, relative to shifting and cancelling of Clifford generators. Since $C$ is a group, either

(i) $p \in D$, a standard form generating set of $C$, or

(ii) $p=\left(g_{1}+g_{2}+\cdots+g_{r}\right)$, where $g_{i} \in D$, for any such $D$.

Assume (i) holds. Then $p=\left(p_{1}, p_{2}, \ldots, p_{t}\right)$ such that $p_{i} \leq$ $(N-k)$ for $i \neq t$, and $p_{t}>(N-k)$, with the first $(N-k)$ edge dimensions defined relative to the particular standard form generating set $D$ being considered. In other words, $p_{t}=$ $p_{1} \cdot p_{2} \cdot \ldots \cdot p_{t-1}$. Then $\forall x \in G, x=\left(x_{1} x_{2}, \ldots, x_{N-k}\right)$, and the sign of $M_{x, x}$, omitting a factor of $(a-1) / 2$, is given by (substituting the formulas for $\pi_{i}(x)$ of Section 3 )

$$
\begin{aligned}
\left(p_{1} \cdot x\right. & \left.+p_{2} \cdot x+\cdots+p_{t-1} \cdot x\right)+x \cdot p_{t} \\
\equiv & p_{2}\left(x_{1}\right)+p_{3}\left(x_{1}+x_{2}\right)+\cdots \\
& +p_{t-1}\left(x_{1}+x_{2}+\cdots+x_{t-2}\right) \\
& +p_{1}\left(x_{2}+\cdots+x_{t-1}\right)+\cdots+p_{t-2}\left(x_{t-1}\right)+|x| \\
\equiv & (|p|-1)|x|-\sum_{i=1}^{t-1}\left(p_{i} x_{i}\right)+|x| \equiv|x||p|+\langle x, p\rangle
\end{aligned}
$$

$(\bmod 2)$.

Note that $|p| \equiv 0(\bmod 4)$, so the first term disappears. The same arguments can be followed to show that this holds for case (ii) also. Then since the factor of $(a-1) / 2$ is constant for all $x \in G$, it follows that, for all $x, y \in G, M_{x, x}=M_{y, y}$ if and only if $\langle x, p\rangle=\langle y, p\rangle$.

One immediate corollary of the preceding lemma is that the trace of $M$ will vanish whenever $P_{M}$ corresponds to a codeword in $C$. In fact, $\operatorname{Tr}(M)$ will only be nonzero in the trivial case where path $P_{M}$ consists of a set of pairs of edges of the same colour. These are the paths corresponding to the Kronecker delta terms of (21). In particular, this implies that $\operatorname{Tr}(M)$ cannot distinguish between equivalence classes of Adinkras directly. However if we instead consider powers of $L$ and $R$ matrices, the preceding lemma suggests a direct generalisation of $(21)$ to all $(N, k)$ Adinkras.

Given an $(N, k)$ Adinkra, consider the product of $t L$ matrices, $L_{i_{1}} L_{i_{2}}^{T} \cdots L_{i_{t}}^{T}$. Denoting path $p=\left(i_{1}, i_{2}, \ldots, i_{t}\right)$, we define value $\sigma_{p}$ such that $\sigma_{p}=0$ if there exists an edge dimension in $p$ that is present $1(\bmod 2)$ times, and $\sigma_{p}=a$ otherwise. Here $a= \pm 1$, corresponding to the sign of the related product of Clifford generators (since $p$ consists of pairs of edges of the same colour, this product of Clifford generators equals \pm 1 ). Then we have the following result.

Lemma 31. The trace of $L_{i_{1}} L_{i_{2}}^{T} \cdots L_{i_{N-1}} L_{i_{N}}^{T}$, where $p=$ $\left(i_{1}, i_{2}, \ldots, i_{N}\right)$, equals

$$
2^{N-k-1}\left(\sigma_{p}+\chi_{0} \epsilon_{p}\right),
$$

where $\chi_{0}= \pm 1$ depending on the equivalence class of the $(N, k)$ valise Adinkras.

Proof. The $\sigma_{p}$ term follows directly from Lemmas 28 and 30 . In Section 4, we show that $(N, k)$ Adinkras with the same associated code $C$ are all in a single equivalence class, except where $(11 \cdots 1) \in C$. In this case, the two equivalence classes are related via the Klein flip operation, exchanging bosons and fermions. Moreover, since $c=(11 \cdots 1) \in C$, the Klein flip operation switches the sign of $\langle x, c\rangle$, for each $x \in V$. Then by Lemma 30, the Adinkras from different equivalence classes correspond to $\chi_{0}$ values of opposite sign.

Note that if $N=4$ and $p=(i, j, k, l)$, then $\sigma_{p}=\delta_{i j} \delta_{k l}$ $\delta_{i k} \delta_{j l}+\delta_{i l} \delta_{j k}$, and the result of (21) follows. These results can 


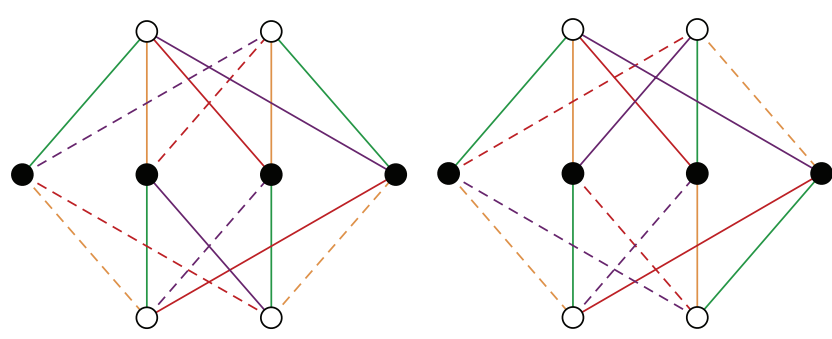

Figure 5: Two equivalent, nonisomorphic Adinkras with the same values of $\mu(h, a)$, for all possible values of $h$ and $a$.

be simplified in some respects if we consider the products of $\gamma$ matrices instead. Recall the definition $M=\gamma_{i_{1}} \gamma_{i_{2}} \cdots \gamma_{i_{t}}$, where $P_{M}=\left(i_{1}, i_{2}, \ldots, i_{t}\right)$, and the fermion number operator $(-1)^{F}$. Instead of taking the trace of $M$, consider the trace of $M$. $(-1)^{F}$. We have

$$
\begin{aligned}
\gamma_{i} & =\left[\begin{array}{cc}
0 & L_{i} \\
R_{i} & 0
\end{array}\right], \\
(-1)^{F} & =\left[\begin{array}{cc}
\mathbb{1} & 0 \\
0 & -\mathbb{1}
\end{array}\right],
\end{aligned}
$$

and we are considering only $L=R^{T}$, so for even $t$,

$$
M=\left[\begin{array}{cc}
L_{i_{1}} L_{i_{2}}^{T} \cdots L_{i_{t}}^{T} & 0 \\
0 & L_{i_{1}}^{T} L_{i_{2}} \cdots L_{i_{t}}
\end{array}\right] .
$$

Replacing $L$ matrices by $R$ matrices in Lemma 31 simply changes the sign of $\chi_{0}$, so for an $(N, k)$ Adinkra,

$$
\operatorname{Tr}\left(M \cdot(-1)^{F}\right)=2^{N-k} \chi_{0} \epsilon_{p} .
$$

\section{Identifying Isomorphism Classes of Adinkras}

The results up to this point deal with equivalence classes of valise Adinkras, where we consider only 2-level Adinkras. For the nonvalise case, we require a method of partitioning general $(N, k)$ Adinkras into their isomorphism classes. The essential problem in establishing such a method is in classifying the topology of an Adinkra relative to the automorphism group of its underlying 1-level Adinkra (in which vertex colouring is ignored). We might consider simply partitioning the vertex set into the orbits of this underlying automorphism group and then classifying each height by the number of vertices of each orbit that it possesses. However this method will clearly be insufficient, as it ignores the relative connectivity between vertices at different heights. For example, the two Adinkras of Figure 5 are in the same equivalence class by use of this set of definitions. They also have the same number of vertices from each orbit at each height, and yet they are clearly nonisomorphic; no relabelling of the vertices or vertex switching operations can map between them. However even this simple method does come close to partitioning nonvalise Adinkras into their isomorphism classes. Recall that a pointwise stabiliser of the automorphism group of any Adinkra is the identity; no nontrivial automorphisms exist that fix any vertex. Note that here the term nontrivial refers to the corresponding permutation of the vertex set. An automorphism which switches vertices but leaves the ordering of the vertex set unchanged is considered trivial. In fact fixing any vertex of an Adinkra yields a natural, canonical ordering of the vertex set, relative to some ordering of the edge dimensions, according to the following construction.

Construction 1. Suppose we are given an $(N, k)$ Adinkra $G=(V, E$, hgt, $\chi, \pi)$, together with an ordering of the edge dimensions of $\left(i_{1}, i_{2}, \ldots, i_{N}\right)$. Then fix (choose) any vertex $v \in V$. We define an ordering $\lambda_{v}$ of the vertex set relative to $v$, where $\lambda_{v}: V \mapsto\left[2^{N-k}\right]$, in the following way.

Consider

(i) $\lambda_{v}(v)=1$.

(ii) Order the neighbours of $v$ from 2 to $N+1$ according to the ordering of the corresponding edge dimensions $\left(\lambda_{v}\left(i_{n} v\right)=1+n\right.$, where $\left.1 \leq n \leq N\right)$.

(iii) Repeat this for vertices at distance 2 , beginning with the neighbours of $i_{1} v$ and ending with the neighbours of $i_{N} v$.

(iv) Repeat this similarly for vertices at each distance, until all vertices have been assigned an ordering in $\left[2^{N-k}\right]$.

In other words, $\lambda_{v}$ firstly orders the vertex set according to distance from $v$; then for each of these sets, each vertex $x$ is assigned an ordering based on the lexicographically smallest path from $v$ to $x$.

To formalise the partitioning of heights discussed above, consider an $(N, k)$ Adinkra $G=(V, E$, hgt, $\chi, \pi)$ with $h$ different height assignments. Let $\Gamma_{G}$ be the automorphism group of the corresponding 1-level Adinkra (ignoring the vertex colouring of $G$ ). Relative to a particular ordering of the edge dimensions, and a particular generating set of the associated doubly even codes, order the $2^{k}$ orbits of $\Gamma_{G}$ according to $\tau: V \mapsto\left[2^{k}\right]$. We then define $\mu_{G}(h, a)$ to be the number of vertices at height $h$ belonging to the $a$ th orbit of $\Gamma_{G}$, such that

$$
\mu_{G}(h, a)=|\{v \in V: \operatorname{hgt}(v)=h, \tau(v)=a\}| .
$$

Consider a pair of Adinkras $G=\left(V, E\right.$, hgt $\left._{1}, \chi_{1}, \pi_{1}\right)$ and $H=$ $\left(V, E^{\prime}\right.$, hgt $\left._{2}, \chi_{2}, \pi_{2}\right)$ belonging to the same equivalence class, both having $t$ distinct heights. Then $G$ and $H$ have the same associated code $C$. If $\mu_{G}(h, a)=\mu_{H}(h, a)$, for all $1 \leq h \leq t$, relative to a given edge-colour ordering and generating set of $C$, then choose any vertex $v \in V$, which when viewed in each Adinkra (as the two Adinkras share the same vertex set) belongs to the same orbit and height. Relative to this vertex $v$, consider the unordered set

$$
\operatorname{cert}_{G}(v)=\left\{\left(\lambda_{v}(x), \operatorname{hgt}(x), \tau(x)\right): x \in V\right\} .
$$

Theorem 32. Consider two Adinkras $G=\left(V, E\right.$, hgt $\left._{1}, \chi_{1}, \pi_{1}\right)$ and $H=\left(V, E^{\prime}\right.$, hgt $\left._{2}, \chi_{2}, \pi_{2}\right)$ and a vertex $v \in V$ with the 


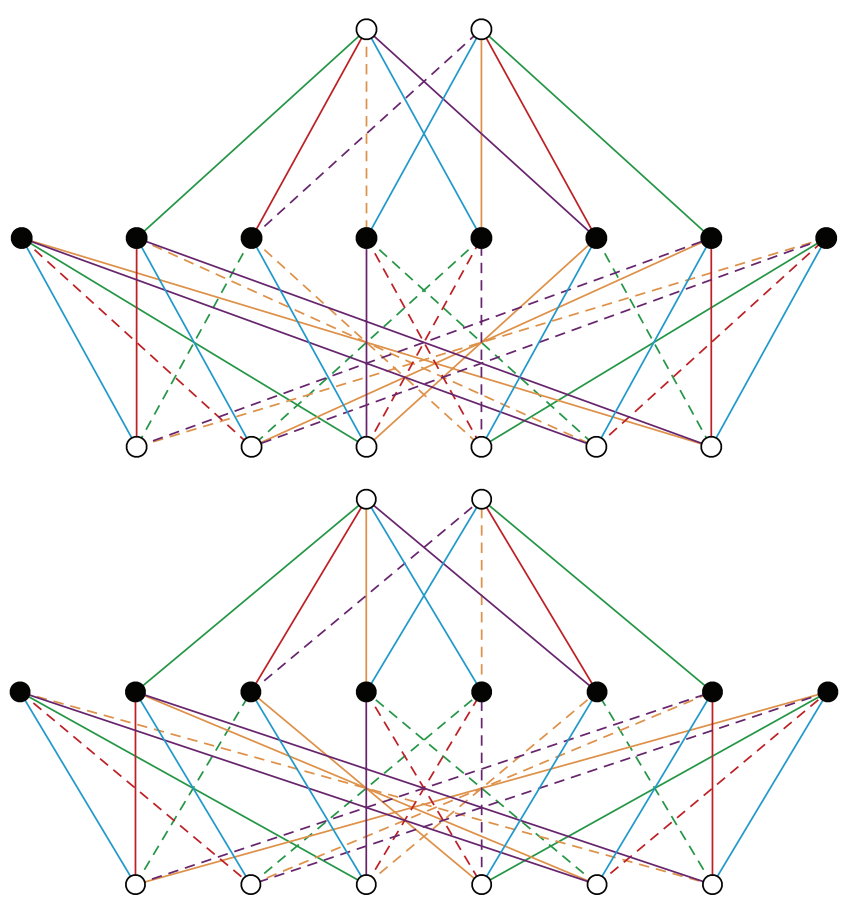

Figure 6: Two equivalent, nonisomorphic $(5,1)$ Adinkras. They differ precisely in the switching state of the yellow edges.

properties described above. Then $\operatorname{cert}_{G}(v)=\operatorname{cert}_{H}(v)$ if and only if $G$ and $H$ are isomorphic.

Proof. If $G$ and $H$ are isomorphic then this is trivially true. Conversely, assume $\operatorname{cert}_{G}(v)=\operatorname{cert}_{H}(v)$. Then since $G$ and $H$ belong to the same equivalence class and $\tau_{G}(v)=\tau_{H}(v)$, there exists an isomorphism $\gamma$ mapping $G$ to $H$ (ignoring the vertex colourings). Then $\gamma$ extends to a full isomorphism (including the vertex colourings) if it preserves height assignments. This follows directly from $\operatorname{cert}_{G}(v)=\operatorname{cert}_{H}(v)$; hence $G$ and $H$ are isomorphic.

The preceding theorem provides an efficient method of classifying Adinkras according to their isomorphism class. Note that the classification is relative to a given ordering of the edge colours and requires a knowledge of the associated doubly even code. In cases where the associated code is unknown, Lemma 27 suggests an efficient method for finding the code and hence relating a given $(N, k)$ Adinkra to its "parent" $N$-cube Adinkra, in the sense of Theorem 9. In particular, Lemma 27 implies the following result.

Corollary 33. Given an $(N, k)$ Adinkra with related code $C$, the codewords of $C$ correspond exactly to the cycles of $G$ in which at least 1 edge dimension appears once (modulo 2).

We provide an example of the above certificates below. Consider the two Adinkras of Figure 6. These are both height $3,(5,1)$ Adinkras. They are in the same equivalence class; however by calculating the above certificate for each we show that they are nonisomorphic.

To analyse the above Adinkras, we first order the edge colours from green to purple, such that, in the codeword representation, green corresponds to the last coordinate and purple to the first. Note that henceforth we will order the right-most coordinate to the left-most coordinate, consistent with a bit-string interpretation of codewords in the computational application. Alternatively, the green edges are associated with the first Clifford generator and the purple edges with the fifth generator. As these are $(5,1)$ Adinkras, they have an associated $(5,1)$ doubly even code. By inspection or by explicitly finding the nontrivial cycles in the Adinkras, we note that the associated code is (11110), corresponding to a 4-cycle comprising edge colours \{red, yellow, blue, purple\}. By Theorem 20, the induced 1-level Adinkra has two orbits, corresponding to the sets of nodes with the same parity (inner product modulo 2) relative to this code. Denote the two Adinkras by $G$ and $H$, respectively. Then by applying the results of Section 4 , we obtain $\mu$ values of

$$
\begin{aligned}
& \mu_{G}(3,1)=2, \\
& \mu_{G}(3,2)=0, \\
& \mu_{G}(2,1)=\mu_{G}(2,2)=4, \\
& \mu_{G}(1,1)=2, \\
& \mu_{G}(1,2)=4, \\
& \mu_{H}(3,1)=0, \\
& \mu_{H}(3,2)=2, \\
& \mu_{H}(2,1)=\mu_{H}(2,2)=4, \\
& \mu_{H}(1,1)=4, \\
& \mu_{H}(1,2)=2 .
\end{aligned}
$$

In other words, the two height 3 vertices of $G$ are in a different orbit to those of $H$. Hence $\operatorname{cert}_{G} \neq \operatorname{cert}_{H}$, and the two Adinkras are in different isomorphism classes.

6.1. Partitioning into Isomorphism Classes: Further Examples. In [20] it was shown that simply recording the number of vertices at each height is not sufficient to characterise Adinkras. In particular, they provide examples of pairs of Adinkras which are in the same equivalence class, but not isomorphic, despite having the same number of vertices at each height. In this section, we analyse the examples of [20] using the techniques described above.

Firstly however, we note that a different definition of isomorphism is considered in [20]. Specifically, they consider the situation where permutations of the edge colours preserve isomorphism, a variation discussed in Section 3.1. Allowing this more general definition of isomorphism results in several changes to the results of the preceding sections. In particular, note that the automorphism group of the $\mathrm{N}$ cube Adinkra would simply become that of the $N$-cube: the hyperoctahedral group of order $2^{N} N$ !. A permutation of the edge colours corresponds to a reordering of the codeword associated with each vertex (or equivalently a permutation of the Clifford generators associated with each edge dimension). 
Hence the automorphism group of $(N, k)$ Adinkras will be extended according to the following lemma.

Lemma 34. Allowing permutations of the edge colours to preserve isomorphism, an $(N, k)$ Adinkra $G$ with associated doubly even code $C$ has an automorphism group (ignoring height assignments) of order

$$
\frac{2^{N}}{2^{2 k+a}}|\operatorname{Aut}(C)|
$$

where Aut $(C)$ is the automorphism group of the code $C$ and where $a=1$ if $C$ contains the all-1 codeword, and $a=0$ otherwise.

In other words, any permutations that correspond to symmetries of the code will extend naturally to automorphisms of the Adinkra. Conversely, if a permutation of the codeword is not in the automorphism group of the code, then trivially it cannot be an automorphism of the Adinkra. Note that Lemma 34 applies trivially to $N$-cube Adinkras, for which $k=0$ and $C=(00 \cdots 0)$; hence $|\operatorname{Aut}(C)|=N$ !.

Hence, Klein flip degeneracy no longer necessarily exhibits for Adinkras containing the all-1 codeword. Instead, note that any automorphism of the associated doubly even code corresponding to an odd permutation of the edge colours (or alternatively the underlying supercharges) effectively performs a Klein flip, substituting bosons for fermions and vice versa. Hence, Klein flip degeneracy occurs if and only if the associated code contains the all-1 codeword and the automorphism group of the code contains no odd permutations, hence narrowing down the possible candidates.

Somewhat fortuitously, a recent result regarding doubly even codes sheds some light on this situation. Doran et al. [20] prove that the automorphism group of self-dual doubly even codes is always contained in the alternating group. Given a doubly even binary $(N, k)$ code $C$, its dual $C^{\perp}$ is given by

$$
C^{\perp}=\left\{v=\left(v_{1}, \ldots, v_{n}\right) \in \mathbb{Z}_{2}^{n}:\langle v, c\rangle=0 \forall c \in C\right\} .
$$

A self-dual code is simply a code equal to its own dual; a doubly even $(N, k)$ code is self-dual if and only if $k=N / 2$ and $N \equiv 0(\bmod 8)$. Hence the following result holds.

Lemma 35. An $(N, k)$ Adinkra exhibits Klein flip degeneracy (relative to this definition of isomorphism, allowing edge-colour permutations) if $N=2 k$ and $N \equiv 0(\bmod 8)$.

However, the question of whether any other Adinkras with Klein flip degeneracy (again allowing for edge-colour permutations) exist still remains. This is equivalent to asking whether any doubly even codes exist, which are both not selfdual and contain no nontrivial even-permutation automorphisms, and is the subject of continuing work. Furthermore, if the automorphism group of doubly even codes turns out to be sufficiently rich, a greatly simplified certificate encoding the isomorphism class of an Adinkra could be developed, as will be discussed briefly in Section 8 .

In [20], two pairs of equivalent but nonisomorphic Adinkras are presented, each pair having the same number
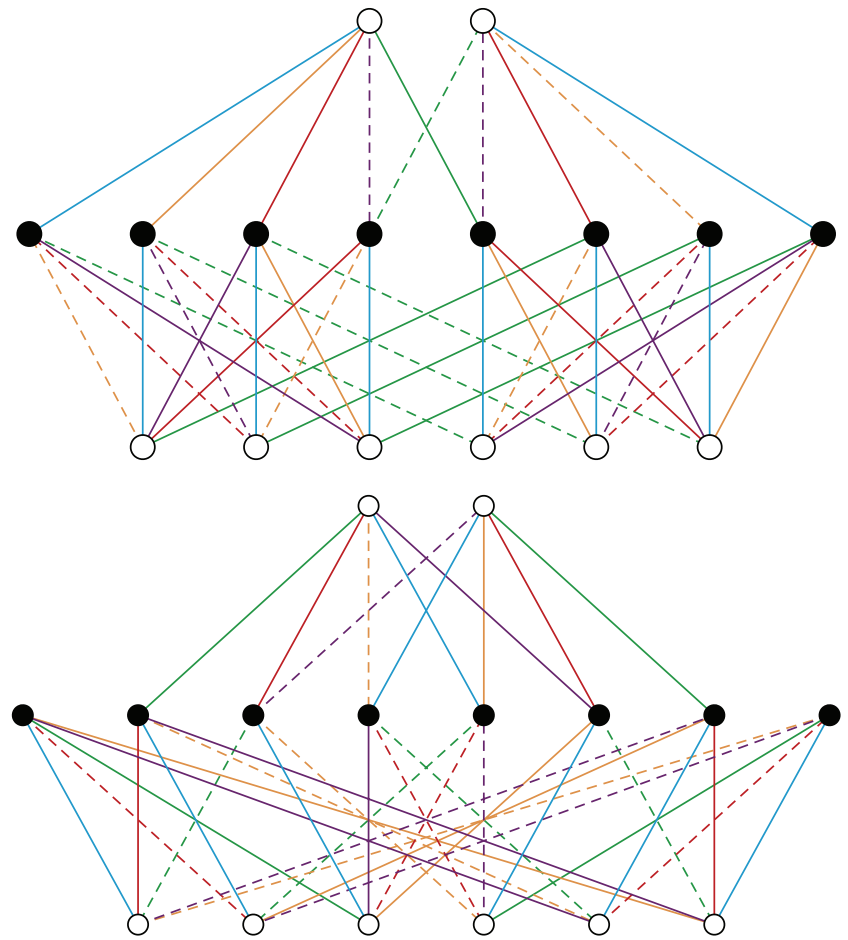

Figure 7: Two nonisomorphic height $3,(5,1)$ Adinkras belonging to the same equivalence class.

of vertices at each height. The first pair comprises two height $3,(5,1)$ Adinkras, isomorphic (up to a permutation of edge colours) to those of Figure 7. Note that the second Adinkra of Figure 7 is identical to the first Adinkra of Figure 6.

Applying the methods described in the previous section to this pair proceeds as in the analysis of the Adinkras of Figure 6. As in that example, after ordering the edge colours from blue to green, each Adinkra has associated code (11110). We see that the top two nodes of the first Adinkra belong to different orbits of the corresponding 1-level Adinkra, whereas the top two nodes of the second Adinkra belong to the same orbit. Hence the two Adinkras are trivially distinguished. In particular, following the conventions in the equations of Example (29), the $\mu$ values of these two Adinkras, labelled by $G$ and $H$, respectively, are

$$
\begin{aligned}
& \mu_{G}(3,1)=1 \\
& \mu_{G}(3,2)=1 \\
& \mu_{G}(2,1)=\mu_{G}(2,2)=4 \\
& \mu_{G}(1,1)=3 \\
& \mu_{G}(1,2)=3 \\
& \mu_{H}(3,1)=2 \\
& \mu_{H}(3,2)=0 \\
& \mu_{H}(2,1)=\mu_{H}(2,2)=4 \\
& \mu_{H}(1,1)=2 \\
& \mu_{H}(1,2)=4 .
\end{aligned}
$$


Hence $\operatorname{cert}_{G} \neq \operatorname{cert}_{H}$, and the two Adinkras are in different isomorphism classes. Also, note that the "orbit spread," the number of vertices in each orbit at each height, has a different character in each Adinkra. So regardless of the ordering of the orbits, these two Adinkras must remain in different isomorphism classes if edge-colour permutations are allowed.

The second pair of Adinkras in [20] comprises two height $3,(6,2)$ Adinkras, isomorphic to the pair displayed in Figure 8.

In this case, after ordering the edge colours from dark blue to green, as for the top left node of the first Adinkra, both

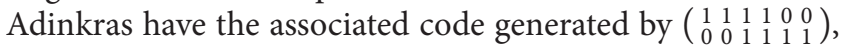
and hence each has four orbits in the automorphism group of the corresponding 1-level Adinkras. The top two nodes of the first Adinkra are connected via the length 2 paths (001001) and (000110), having odd inner product with each of the codewords in the generating set above. Hence these nodes are in different orbits of the automorphism group. Conversely, the top two nodes of the second Adinkra are connected via the length 2 paths (110000), (001100), and (000011), having even inner product with each of the codewords. Hence they are in the same orbit. As a result, the certificates of Theorem 32 are different for each Adinkra, and hence they are in different isomorphism classes. Again, following the conventions in the equations of Example (29), we obtain $\mu$ values of

$$
\begin{aligned}
& \mu_{G}(3,1)=\mu_{G}(3,2)=1 \\
& \mu_{G}(2, i)=2, \quad \forall i \in[4] \\
& \mu_{G}(1,1)=\mu_{G}(1,2)=1 \\
& \mu_{G}(1,3)=\mu_{G}(1,4)=2 \\
& \mu_{H}(3,2)=2 \\
& \mu_{H}(2, i)=2, \quad \forall i \in[4] \\
& \mu_{H}(1,2)=0 \\
& \mu_{H}(1,1)=\mu_{G}(1,3)=\mu_{G}(1,4)=2 .
\end{aligned}
$$

Hence, as in the previous example, $\operatorname{cert}_{G} \neq \operatorname{cert}_{H}$, and moreover the Adinkras remain in different isomorphism classes if edge-colour permutations are allowed.

\section{Numerical Results}

The automorphism group results of the preceding sections were also verified numerically, independently to the analytical results. All $(N, k)$ Adinkras up to $N \leq 16$ were produced, and the related automorphism group and equivalence classes were calculated for each such Adinkra. The results found were consistent with the analytical results described in the earlier sections. In particular,

(i) all $(N, k)$ Adinkras with the same associated code $C$ were found to be in the same equivalence class, except in the cases where $(11 \cdots 1) \in C$. In these cases, the Adinkras were split into two equivalence classes,

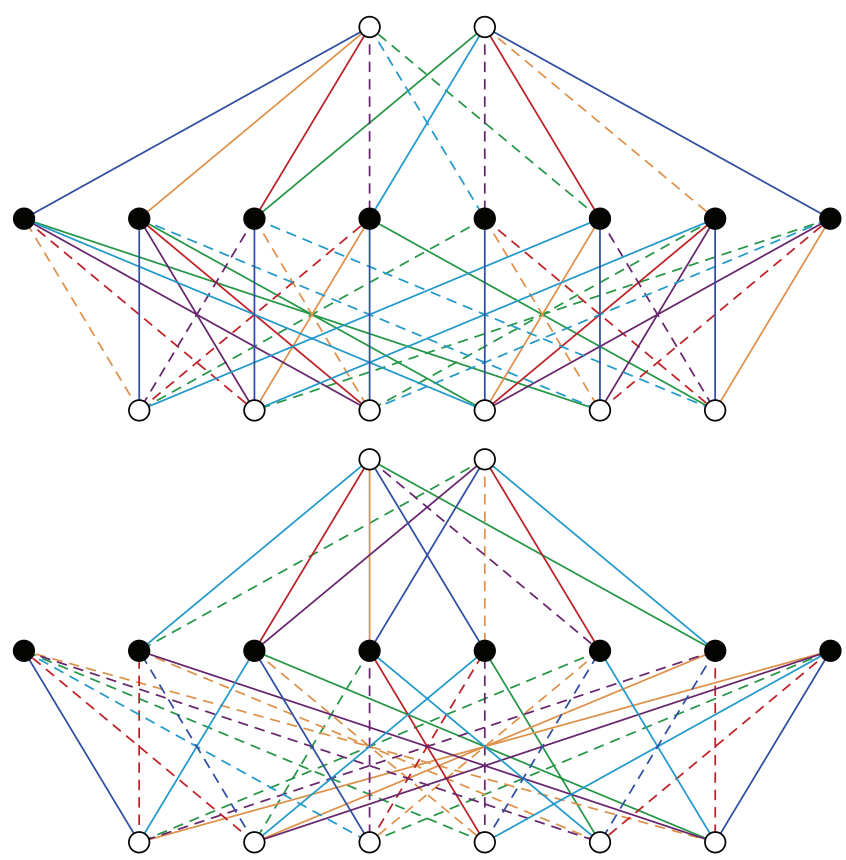

Figure 8: Two nonisomorphic height 3, $(6,2)$ Adinkras belonging to the same equivalence class.

related via the Klein flip operation, as described in Corollary 22;

(ii) all 1-level $(N, k)$ Adinkras had $2^{k}$ orbits, each consisting of sets of vertices with the same set of inner products with the codewords in $C$, according to Theorem 20.

All doubly even $(N, k)$ codes up to $N=28$ (and many larger parameter sets) can be found online (Miller, [21]). For each parameter set $(N, k)$, the two possibly inequivalent standard form Adinkras corresponding to the construction method of Theorem 14 were produced relative to each doubly even code on these parameters. As we were considering only equivalence classes and automorphism groups of valise Adinkras, it sufficed to use the adjacency matrix form defined in Section 5 for this analysis, as height assignments need not be encoded in the representation. The orbits were then calculated from the adjacency matrices by forming a canonical form relative to each node via a set of switching operations and a permutation of the vertex set. A canonical form is defined to be a mapping $\pi$, consisting of a permutation of the vertex labels and set of vertex switching operations, such that for any two Adinkras $G$ and $H, \pi(G)=\pi(H)$ if and only if $G$ and $H$ are isomorphic.

Then given a vertex $v$ and adjacency matrix $A$ of an $(N, k)$ Adinkra (together with an ordering of the edge colours), we define the following canonical form of $A$ relative to $v$.

(i) Permute the ordering of the vertices relative to their connections to $v$ and the ordering of edge colours, such that the vertices are ordered:

$$
\begin{aligned}
& \left(v, i_{1} v, \ldots, i_{N} v, i_{2} i_{1} v, \ldots, i_{N} i_{1} v, i_{3} i_{2} v, \ldots, i_{N} i_{N-1}\right. \\
& \left.\quad \ldots i_{1} v\right) .
\end{aligned}
$$




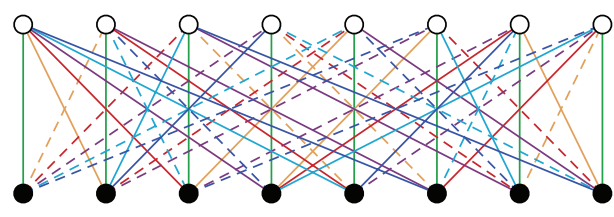

FIGURE 9: Standard form valise Adinkra.

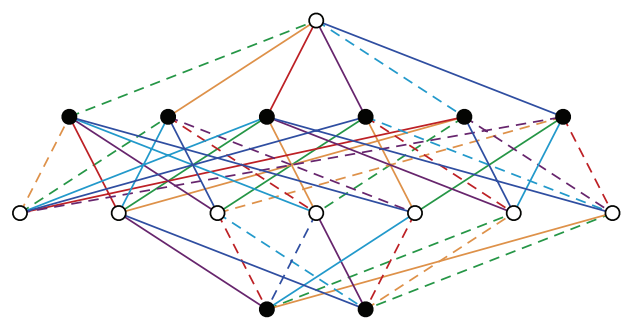

Figure 10: Adinkra after step (i).

(ii) Switch vertices $\left(i_{1} v, \ldots, i_{N} v\right)$ such that all edges of $v$ have even parity.

(iii) Repeat for neighbours of vertex $i_{1} v$.

(iv) Repeat for each vertex, in the ordering above, such that edges appearing lexicographically earlier in the adjacency matrix are of even parity where possible.

This defines a unique switching state of the Adinkra, up to isomorphism. Furthermore it is a canonical form, in that any two vertices belonging to the same orbit result in identical matrix forms. To illustrate the above process, we consider a $(6,2)$ Adinkra with associated code generated by $\left(\begin{array}{llllll}1 & 0 & 1 & 1 & 1 & 0 \\ 0 & 1 & 1 & 1 & 0 & 1\end{array}\right)$. The standard form valise Adinkra is shown in Figure 9, where the top leftmost vertex has codeword (0000), and its edges are ordered lexicographically from left to right.

Consider the process of converting this Adinkra to canonical form, relative to vertex (0011) (the fifth white vertex from the left in Figure 9). If we order the vertices by height first, then from left to right, step (i) corresponds to the set of vertex lowering operations leading to the Adinkra shown in Figure 10.

Steps (ii) and (iii) correspond to a set of switching operations, permuting the switching state in the following stages, as shown in Figure 11.

Step (iv) then involves the remaining set of transformations, shown in Figure 12.

At this point the Adinkra is now in canonical form. The particular switching state of this resulting Adinkra is unique to choices of source vertices in the same orbit, in this case the set of vertices (0000), (0011), (1110), (1101).

\section{Conclusions and Future Work}

This work provides a graph theoretic characterisation of Adinkras, in particular classifying their automorphism groups according to an efficiently computable set of local parameters. In the current work, a number of comparisons are made to previous work. The connection between Adinkras and codes [4] has been reexamined and found to be robust. However, this connection is also exploited to utilise the standard form leading to more computationally efficient algorithms for the study of Adinkras. As well, the observations based on matrix methods used within the context of $d=N=4$ [5] have now been extended by a formal proof to all values of $d$ and $N$. Also as emphasised in Section 7, numerical studies up to values of $N=16$ provide additional concurrence. These results support the proposal that $\chi_{0}$ "chi-null" is a class valued function defined on valise Adinkras.

All nonvalise Adinkras through a series of node raising and lowering can be brought to the form of a valise Adinkra. In this sense $\chi_{0}$ is defined for all Adinkras. However, for nonvalise Adinkras, $\chi_{0}$ is not sufficient to define classes. For this purpose, the new certificate $\mu_{\mathscr{A}}(h, a)$, where $\mathscr{A}$ is an arbitrary Adinkra, seems to fill in a missing gap.

Additionally, the classification method used here is robust in the sense that it can be readily adapted to address a variety of definitions of isomorphism/equivalence, relevant to the particular supersymmetric system of interest. For instance, in Section 6.1 the generalised definition of isomorphism including edge-colour permutations yields a generalised certificate.

8.1. Open Questions. It is the work of future investigations to explore whether these tools $\left(\chi_{0}\right.$ and $\left.\mu_{\mathscr{A}}(h, a)\right)$ are sufficient to attack the problem of the complete classification of onedimensional off-shell supersymmetrical systems. One obvious future avenue of study is to investigate the role codes play in Gnomon Adinkras [19] and Escheric Adinkras [1]. This, as part of continuing to attack the general problem, presents continuing challenges.

Note that when edge-colour permutations are allowed, the calculation of the certificate encoding the isomorphism class of an Adinkra, as detailed in Section 6, is currently more complex, compared to the situation when edge-colour permutations do not preserve isomorphism in that the automorphism group of the doubly even code must also be calculated. One future direction of research is the investigation of the properties of these automorphism groups. It is already known that self-dual doubly even codes have automorphism groups contained in the alternating group [22]; however further properties must be discovered to determine all possible Klein flip degenerate Adinkras in this case.

\section{Conflict of Interests}

The authors declare that there is no conflict of interests regarding the publication of this paper.

\section{Acknowledgment}

The authors would like to thank Akihiro Munemasa for detailed comments and suggestions on this paper, as well as Cheryl Praeger, Cai-Heng Li, Gordon Royle, and John Bamberg for several useful discussions. This work was partially supported by the National Science Foundation Grant PHY13515155. The authors would also like to thank the Institute of Advanced Studies at The University of Western Australia for 


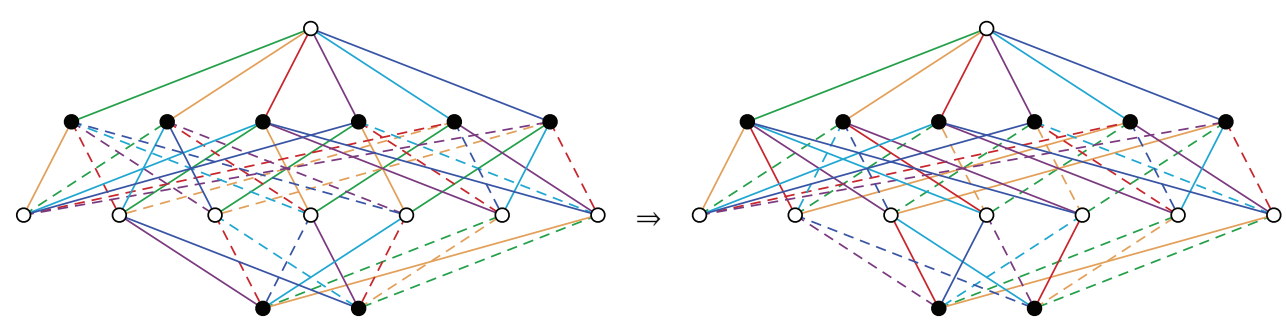

FIGURE 11: Adinkra after steps (ii) and (iii).

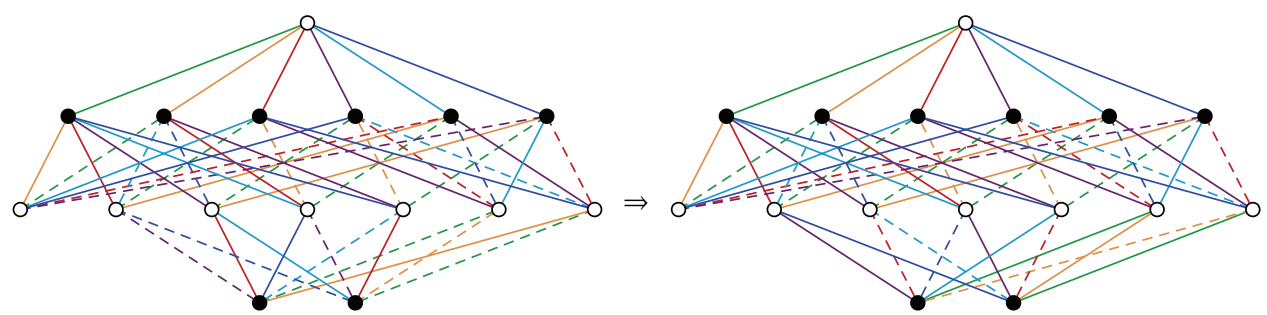

Figure 12: Adinkra after step (iv).

providing travel support, which enabled initial discussions among the authors.

\section{References}

[1] M. Faux and S. J. Gates Jr., "Adinkras: a graphical technology for supersymmetric representation theory," Physical Review D, vol. 71, Article ID 065002, 2005.

[2] C. F. Doran, M. G. Faux, S. J. Gates Jr. et al., "Topology types of adinkras and the corresponding representations of $\mathrm{N}$-extended supersymmetry," http://arxiv.org/abs/0806.0050.

[3] C. F. Doran, M. G. Faux, S. J. Gates Jr. et al., "Adinkras for clifford algebras, and worldline supermultiplets," http://arxiv.org/ abs/0811.3410.

[4] C. F. Doran, M. G. Faux, S. J. Gates Jr. et al., "Relating doublyeven error-correcting codes, graphs, and irreducible representations of $\mathrm{N}$-extended supersymmetry," http://arxiv.org/ abs/0806.0051.

[5] S. J. Gates Jr., J. Gonzales, B. MacGregor et al., " $4 \mathrm{D}, N=1$ supersymmetry genomics (I)," Journal of High Energy Physics, vol. 2009, no. 12, article 008, 2009.

[6] M. G. Faux, S. J. Gates Jr., and T. Hübsch, "Effective symmetries of the minimal supermultiplet of the $N=8$ extended worldline supersymmetry," Journal of Physics A: Mathematical and Theoretical, vol. 42, no. 41, Article ID 415206, 2009.

[7] M. G. Faux, K. M. Iga, and G. D. Landweber, "Dimensional enhancement via supersymmetry," Advances in Mathematical Physics, vol. 2011, Article ID 259089, 45 pages, 2011.

[8] C. F. Doran, M. G. Faux, S. J. Gates Jr., T. Hubsch, K. M. Iga, and G. D. Landweber, "On graph-theoretic identifications of adinkras, supersymmetry representations and superfields," International Journal of Modern Physics A, vol. 22, no. 5, pp. 869930, 2007.

[9] K. Burghardt and S. J. Gates Jr., "Adinkra isomorphisms and 'seeing' shapes with eigenvalues," http://arxiv.org/abs/1212.2731.

[10] K. Burghardt and S. J. Gates Jr., "A computer algorithm for engineering off-shell multiplets with four supercharges on the world sheet," http://arxiv.org/abs/1209.5020.
[11] S. Naples, Classification of Adinkra graphs, http://math.bard .edu/student/pdfs/sylvia-naples.pdf.

[12] S. J. Gates Jr. and L. Rana, "A theory of spinning particles for large N-extended supersymmetry," Physics Letters B, vol. 352, no. 1-2, pp. 50-58, 1995.

[13] S. J. Gates Jr. and L. Rana, "A theory of spinning particles for large $N$-extended supersymmetry (II)," Physics Letters B, vol. 369, no. 3-4, pp. 262-268, 1996.

[14] S. J. Gates Jr., W. D. Linch, J. Phillips, and L. Rana, "The fundamental supersymmetry challenge remains," Gravitation and Cosmology, vol. 8, no. 1, pp. 96-100, 2002.

[15] S. J. Gates Jr., W. D. Linch, and J. Phillips, "When superspace is not enough," http://arxiv.org/abs/hep-th/0211034.

[16] C. Godsil and G. Royle, Algebraic Graph Theory, vol. 207 of Graduate Texts in Mathematics, Springer, New York, NY, USA, 2001.

[17] A. Brouwer and W. Haemers, Spectra of Graphs, Springer, 2012.

[18] J. van Lint and J. Seidel, "Equilateral point sets in elliptic geometry," Indagationes Mathematicae, vol. 28, pp. 335-348, 1966.

[19] C. F. Doran, M. G. Faux, S. J. Gates Jr., T. Hubsch, K. M. Iga, and G. D. Landweber, "Adinkras and the dynamics of superspace prepotentials," http://arxiv.org/abs/hep-th/0605269.

[20] C. F. Doran, M. G. Faux, S. J. Gates Jr., T. Hubsch, K. M. Iga, and G. D. Landweber, "A counter-example to a putative classification of 1-dimensional, N-extended supermultiplets," Advanced Studies in Theoretical Physics, vol. 2, article 99, 2008.

[21] R. L. Miller, "Doubly even codes," http://www.rlmiller.org/de_ codes/.

[22] A. Günther and G. Nebe, "Automorphisms of doubly even selfdual binary codes," Bulletin of the London Mathematical Society, vol. 41, no. 5, pp. 769-778, 2009. 


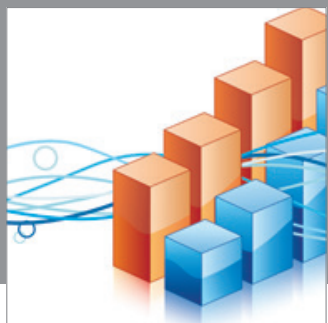

Advances in

Operations Research

mansans

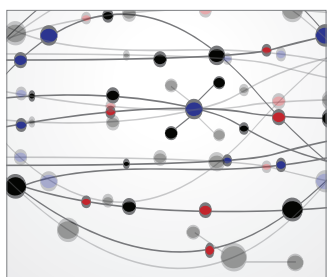

The Scientific World Journal
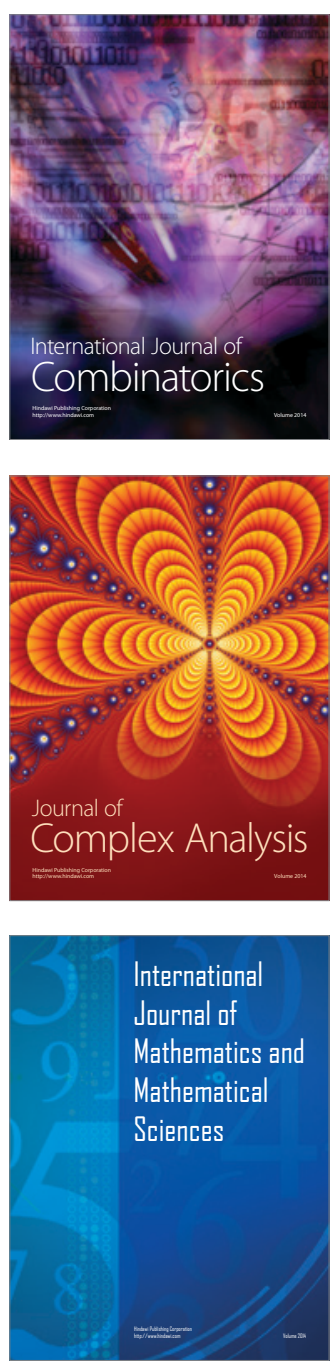
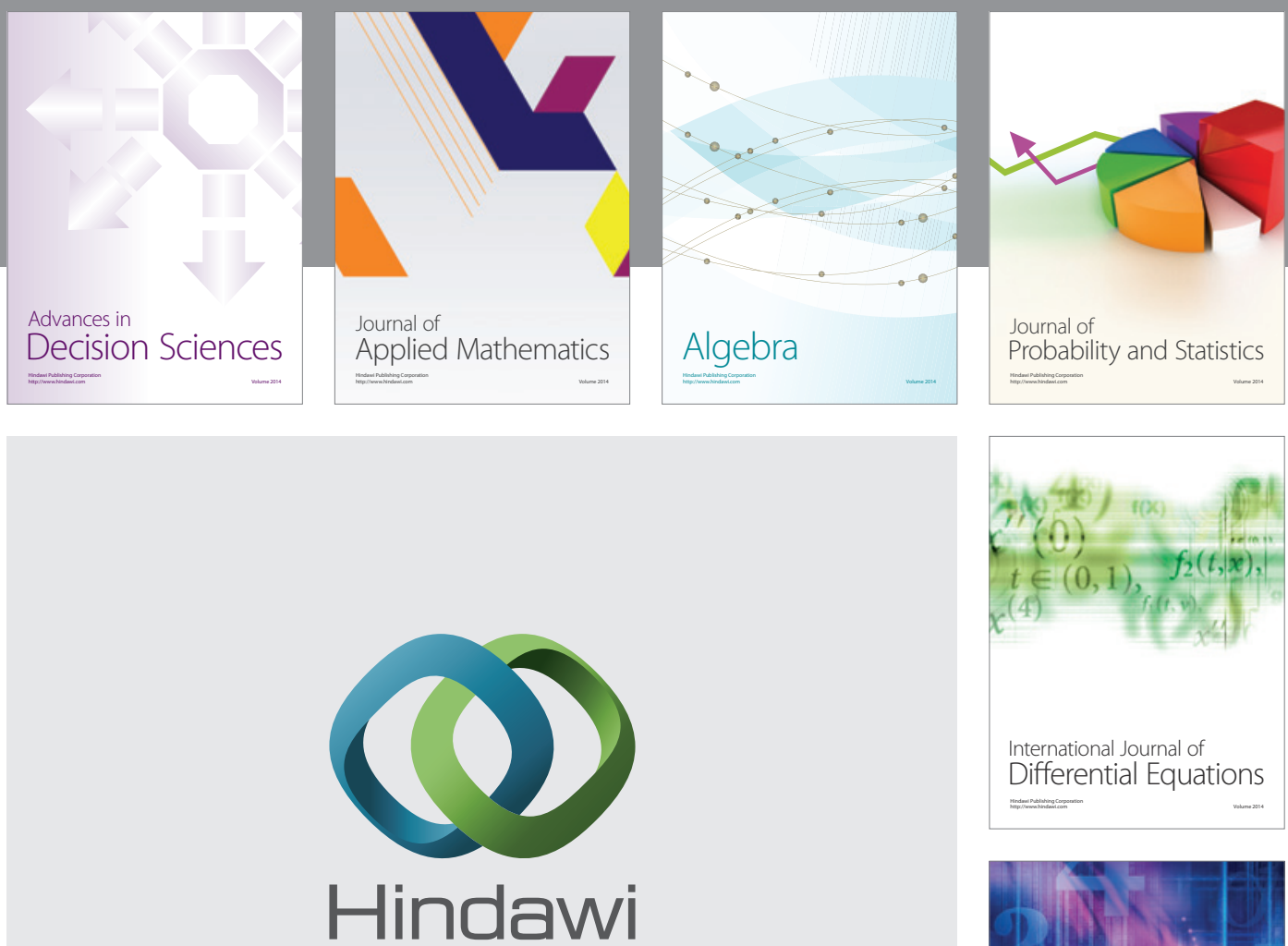

Submit your manuscripts at http://www.hindawi.com
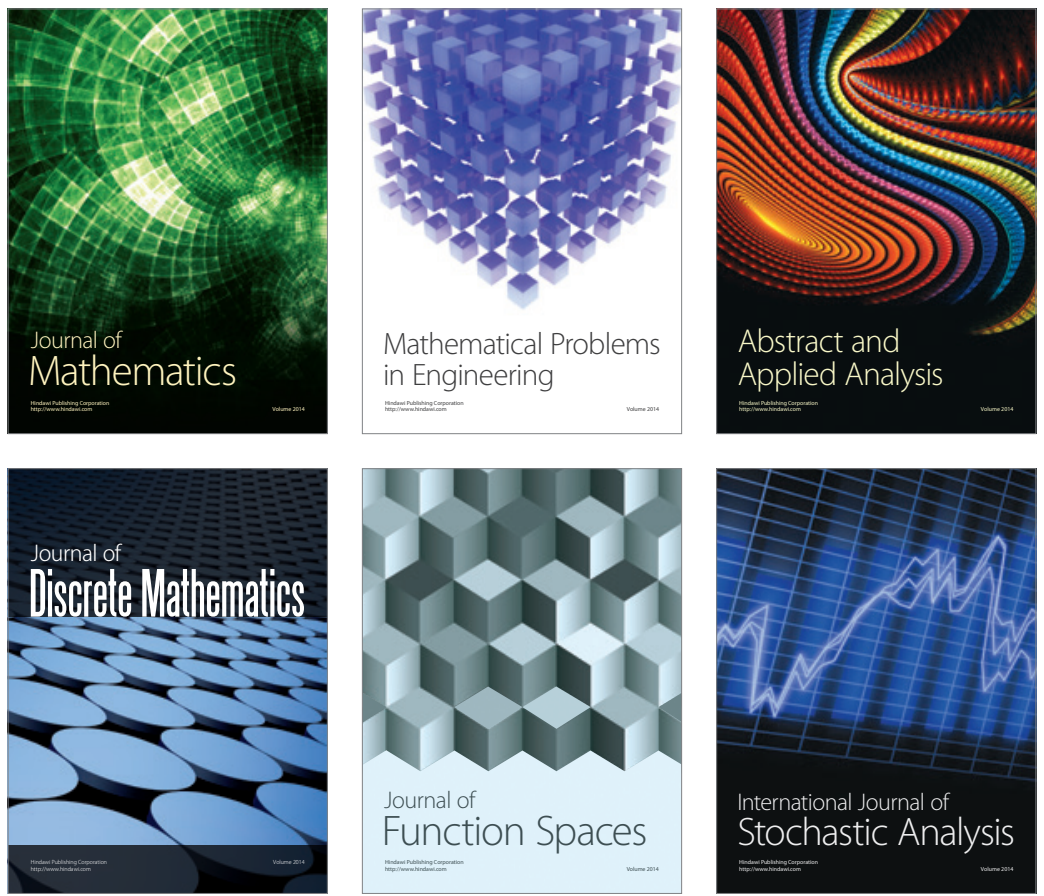

Journal of

Function Spaces

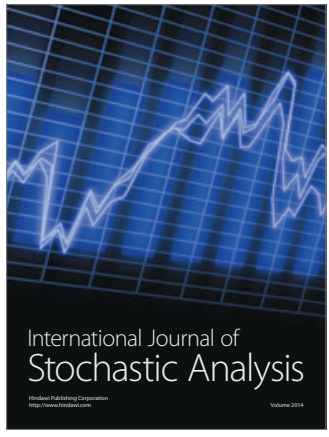

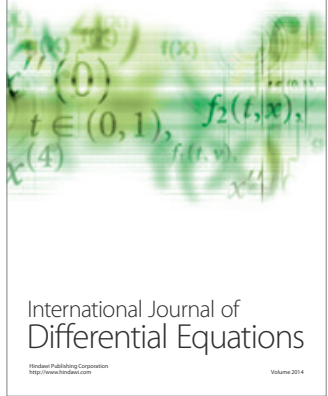
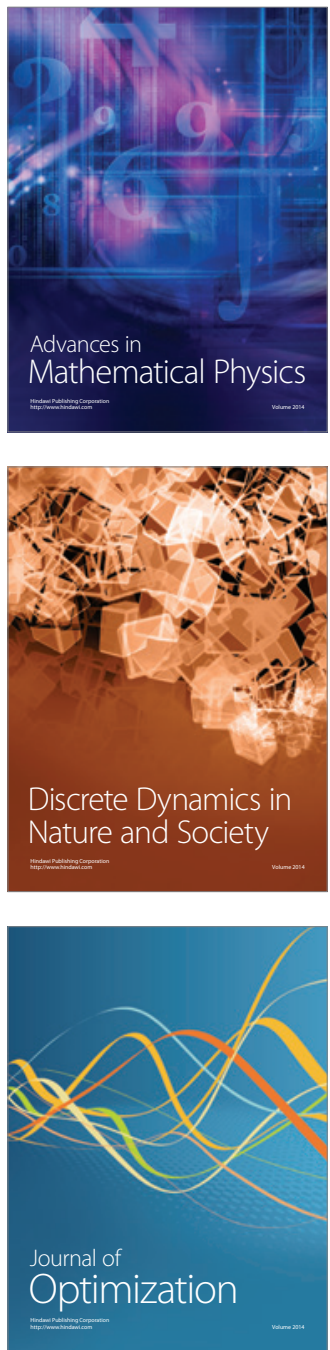